\title{
Consideration and the Refinement of Some Laws and Concepts of Classical Electrodynamics and New Ideas in Modern Electrodynamics
}

\author{
F.F. Mende* \\ B.I. Verkin Institute for Low Temperature Physics and Engineering, NAS Ukraine, 47 Lenin Ave., Kharkov, 61164, Ukraine \\ *Corresponding author: mende_fedor@mail.ru
}

Received September 24, 2014; Revised October 15, 2014; Accepted November 21, 2014

\begin{abstract}
The problems considered refer to the material equations of electromagnetic and magnetoelectric induction and physical interpretation of the parameters $\varepsilon(\omega)$ and $\mu(\omega)$. Some contradictions found in fundamental studies on classical electrodynamics have been explained. The notion magnetoelectric induction has been introduced, which permits symmetrical writing of the induction laws. It is shown that the results of the special theory of relativity can be obtained from these laws through the Galileo conversions with the accuracy to the $\frac{v^{2}}{c^{2}}$ terms. The permittivity and permeability of materials media are shown to be independent of frequency. The notions magnetoelectrokinetic and electromagnetopotential waves and kinetic capacity have been introduced. It is shown that along with the longitudinal Langmuir resonance, the transverse resonance is possible in nonmagnetized plasma, and both the resonances are degenerate. A new notion scalar-vector potential is introduced, which permits solution of all present-day problems of classical electrodynamics. The use of the scalar-vector potential makes the magnetic field notion unnecessary.
\end{abstract}

Keywords: classical electrodynamics, Faraday low, Maxwell questions, electromagnetic induction, Lorentz force, scalar-vector potential, phase aberration, plasma media, dielectric media, magnetic media, kinetic inductivity, polarization vector, London equation, magnetic resonance, magnetoelectrokinetic wave, electromagnetopotential waves, kinetic capacitance

Cite This Article: F.F. Mende, "Consideration and the Refinement of Some Laws and Concepts of Classical Electrodynamics and New Ideas in Modern Electrodynamics." International Journal of Physics, vol. 2, no. 6 (2014): 231-263. doi: 10.12691/ijp-2-6-8.

\section{Introduction}

The laws of classical electrodynamics they reflect experimental facts they are phenomenological. Unfortunately, contemporary classical electrodynamics is not deprived of the contradictions, which did not up to now obtain their explanation.

The fundamental equations of contemporary classical electrodynamics are the Maxwell equation. They are written as follows for the vacuum:

$$
\begin{aligned}
& \operatorname{rot} \vec{E}=-\frac{\partial \vec{B}}{\partial t}, \\
& \operatorname{rot} \vec{H}=\frac{\partial \vec{D}}{\partial t}, \\
& \operatorname{div} \vec{D}=0, \\
& \operatorname{div} \vec{B}=0,
\end{aligned}
$$

where $\vec{E}$ and $\vec{H}$ are tension of electrical and magnetic field, $\vec{D}=\varepsilon_{0} \vec{E}$ and $\vec{B}=\mu_{0} \vec{H}$ are electrical and magnetic induction, $\mu_{0}$ and $\varepsilon_{0}$ are magnetic and dielectric constant of vacuum. From Maxwell equations follow the wave equations

$$
\begin{aligned}
& \nabla^{2} \vec{E}=\mu_{0} \varepsilon_{0} \frac{\partial^{2} \vec{E}}{\partial t^{2}}, \\
& \nabla^{2} \vec{H}=\mu_{0} \varepsilon_{0} \frac{\partial^{2} \vec{H}}{\partial t^{2}} .
\end{aligned}
$$

These equations show that in the vacuum can be extended the plane electromagnetic waves, the velocity of propagation of which is equal to the speed of light

$$
c=\frac{1}{\sqrt{\mu_{0} \varepsilon_{0}}} .
$$

For the material media the Maxwell equations take the following form:

$$
\begin{gathered}
\operatorname{rot} \vec{E}=-\tilde{\mu} \mu_{0} \frac{\partial \vec{H}}{\partial t}=-\frac{\partial \vec{B}}{\partial t}, \\
\operatorname{rot} \vec{H}=n e \vec{v}+\tilde{\varepsilon} \varepsilon_{0} \frac{\partial \vec{E}}{\partial t}=n e \vec{v}+\frac{\partial \vec{D}}{\partial t},
\end{gathered}
$$




$$
\begin{aligned}
& \operatorname{div} \vec{D}=n e, \\
& \operatorname{div} \vec{B}=0,
\end{aligned}
$$

where $\tilde{\mu}$ and $\tilde{\varepsilon}$ are the relative magnetic and dielectric constants of the medium and $n, e$ and $\vec{v}$ are density, value and charge rate.

The equations $(1.1$ - 1.11) are written in the assigned inertial reference frame (IRF) and in them there are no rules of passage of one IRF to another. These equations assume that the properties of charge do not depend on their speed.

In Maxwell equations are not contained indication that is the reason for power interaction of the current-carrying systems, therefore to be introduced the experimental postulate about the force, which acts on the moving charge in the magnetic field

$$
\vec{F}_{L}=e\left[\vec{v} \times \mu_{0} \vec{H}\right] .
$$

This force is called the Lorentz force. However in this axiomatics is an essential deficiency. If force acts on the moving charge, then in accordance with third Newton law must occur and reacting force. In this case the magnetic field is independent substance, comes out in the role of the mediator between the moving charges. Consequently, we do not have law of direct action, which would give immediately answer to the presented question, passing the procedure examined. I.e. we cannot give answer to the question, where are located the forces, the compensating action of magnetic field to the charge.

The equation (1.12) from the physical point sight causes bewilderment. The forces, which act on the body in the absence of losses, must be connected either with its acceleration, if it accomplishes forward motion, or with the centrifugal forces, if body accomplishes rotary motion. Finally, static forces appear when there is the gradient of the scalar potential of potential field, in which is located the body. But in Eq. (1.12) there are no such forces. Usual rectilinear motion causes the force, which is normal to the direction motion. In the classical mechanics the forces of this type are unknown.

Is certain, magnetic field is one of the important concepts of contemporary electrodynamics. Its concept consists in the fact that around any moving charge appears the magnetic field (Ampere law), whose circulation is determined by equation

$$
\oint \vec{H} d \vec{l}=I,
$$

where $I$ is conduction current. Equation (1.9) is the consequence of Eq. (1.13), if we to the conduction current add bias current.

Let us especially note that the introduction of the concept of magnetic field does not be founded upon any physical basis, but it is the statement of the collection of some experimental facts. Using this concept, it is possible with the aid of the specific mathematical procedures to obtain correct answer with the solution of practical problems. But, unfortunately, there is a number of the physical questions, during solution of which within the framework the concepts of magnetic field, are obtained paradoxical results. Here one of them.

Using Eqs. (1.12) and (1.13) not difficult to show that with the unidirectional parallel motion of two like charges, or flows of charges, between them must appear the additional attraction. However, if we pass into the inertial system, which moves together with the charges, then there magnetic field is absent, and there is no additional attraction. This paradox does not have an explanation.

Of force with power interaction of material structures, along which flows the current, are applied not only to the moving charges, but to the lattice, but in the concept of magnetic field to this question there is no answer also, since. In Eqs. (1.1-1.13) the presence of lattice is not considered. At the same time, when current flows through the plasma, occurs its compression. This phenomenon is called pinch effect. In this case forces of compression act not only on the moving electrons, but also on the positively charged ions. And, again, the concept of magnetic field cannot explain this fact, since in this concept there are no forces, which can act on the ions of plasma.

As the fundamental law of induction in the electrodynamics is considered Faraday law, consequence of whom is the Maxwell first equation. However, here are problems. It is considered Until now that the unipolar generator is an exception to the rule of flow, consequently Farrday law is not complete.

Let us give one additional statement of the monograph [1]: "The observations of Faraday led to the discovery of new law about the connection of electrical and magnetic field on: in the field, where magnetic field changes in the course of time, is generated electric field". But from this law also there is an exception. Actually, the magnetic fields be absent out of the long solenoid; however, electric fields are generated with a change of the current in this solenoid around the solenoid. In the classical electrodynamics does not find its explanation this well known physical phenomenon, as phase aberration of light.

From entire aforesaid it is possible to conclude that in the classical electrodynamics there is number of the problems, which still await their solution.

\section{PART I. Consideration and the Refinement of Some Laws and Concepts of Classical Electrodynamics}

\section{Laws of the Magnetoelectric Induction}

The primary task of induction is the presence of laws governing the appearance of electrical field on, since only electric fields exert power influences on the charge.

Faraday law is written as follows:

$$
\text { of } \oint \vec{E} d \vec{l}=-\frac{\partial \Phi_{B}}{\partial t}=-\mu \int \frac{\partial \vec{H}}{\partial t} d \vec{s}=-\int \frac{\partial \vec{B}}{\partial t} d \vec{s} \text {, }
$$

where $\vec{B}=\mu \vec{H}$ is magnetic induction vector, $\Phi_{B}=\mu \int \vec{H} d \vec{s}$ is flow of magnetic induction, and $\mu=\tilde{\mu} \mu_{0}$ - magnetic permeability of medium. It follows from this law that the circulation integral of the vector of electric field is equal to a change in the flow of magnetic induction through the area, which this outline covers. From Eq. (2.1) obtain the Maxwell first equation 


$$
\text { rot } \vec{E}=-\frac{\partial \vec{B}}{\partial t} .
$$

Let us immediately point out to the terminological error. Faraday law should be called not the law of electromagnetic, as is customary in the existing literature, but by the law of magnetoelectric induction, since. a change in the magnetic field on it leads to the appearance of electrical field on, but not vice versa.

Let us introduce the vector potential of the magnetic field $\vec{A}_{H}$, which satisfies the equality

$$
\mu \oint \vec{A}_{H} d \vec{l}=\Phi_{B},
$$

where the outline of the integration coincides with the outline of integration in Eq. (2.1), and the vector of is determined in all sections of this outline, then then

$$
\vec{E}=-\mu \frac{\partial \vec{A}_{H}}{\partial t} .
$$

Between the vector potential and the electric field there is a local connection. Vector potential is connected with the magnetic field with the following equation:

$$
\text { rot } \vec{A}_{H}=\vec{H} \text {. }
$$

During the motion in the three-dimensional changing field of vector potential the electric fields find, using total derivative.

$$
\vec{E}^{\prime}=-\mu \frac{d \vec{A}_{H}}{d t}
$$

Prime near the vector $\vec{E}$ means that we determine this field in the moving coordinate system. This means that the vector potential has not only local, but also convection derivative. In this case Eq. (2.5) can be rewritten as follows:

$$
\vec{E}^{\prime}=-\mu \frac{\partial \vec{A}_{H}}{\partial t}-\mu(\vec{v} \nabla) \vec{A}_{H},
$$

where $\vec{v}$ is speed of system. If vector potential on time does not depend, the force acts on the charge

$$
\vec{F}_{v, 1}^{\prime}=-\mu e(\vec{v} \nabla) \vec{A}_{H} .
$$

This force depends only on the gradients of vector potential and charge rate.

The charge, which moves in the field of the vector potential $\vec{A}_{H}$ with the speed $\vec{v}$, possesses potential energy [1]

$$
W=-e \mu\left(\vec{v} \vec{A}_{H}\right) .
$$

Therefore must exist one additional force, which acts on the charge in the moving coordinate system, namely:

$$
\vec{F}_{v, 2}^{\prime}=-\operatorname{grad} W=e \mu \operatorname{grad}\left(\vec{v} \vec{A}_{H}\right) .
$$

The value $e \mu\left(v \vec{A}_{H}\right)$ plays the same role, as the scalar potential of the charge, whose gradient determines the force, which acts on the moving charge. Consequently, the composite force, which acts on the charge, which moves in the field of vector potential, can have three components and will be written down as

$$
\vec{F}^{\prime}=-e \mu \frac{\partial \vec{A}_{H}}{\partial t}-e \mu(\vec{v} \nabla) \vec{A}_{H}+e \mu \operatorname{grad}\left(\vec{v} \vec{A}_{H}\right) .
$$

The first of the components of this force acts on the fixed charge, when vector potential changes in the time and has local time derivative. Second component is connected with the motion of charge in the threedimensional changing field of this potential. Entirely different nature in force, which is determined by last term Eq. (2.6). It is connected with the fact that the charge, which moves in the field of vector potential, it possesses potential energy, whose gradient gives force. From Eq. (2.6) follows

$$
\vec{E}^{\prime}=-\mu \frac{\partial \vec{A}_{H}}{\partial t}-\mu(\vec{v} \nabla) \vec{A}_{H}+\mu \operatorname{grad}\left(\vec{v} \vec{A}_{H}\right) .
$$

This is a complete law of mutual induction. It defines all electric fields, which can appear at the assigned point of space, this point can be both the fixed and that moving. This united law includes and Faraday law and that part of the Lorentz force, which is connected with the motion of charge in the magnetic field, and without any exceptions gives answer to all questions, which are concerned mutual magnetoelectric induction. It is significant, that, if we take rotor from both parts of equality (2.7), attempting to obtain the Maxwell first equation, then it will be immediately lost the essential part of the information, since. rotor from the gradient is identically equal to zero.

If we isolate those forces, which are connected with the motion of charge in the three-dimensional changing field of vector potential, and to consider that

$$
\mu \operatorname{grad}\left(\vec{v} \vec{A}_{H}\right)-\mu(\vec{v} \nabla) \vec{A}_{H}=\mu\left[\vec{v} \times \operatorname{rot} \vec{A}_{H}\right],
$$

that from Eq. (2.6) we will obtain

$$
\vec{F}_{v}^{\prime}=e \mu\left[\vec{v} \times \operatorname{rot} \vec{A}_{H}\right],
$$

and, taking into account (2.4), let us write down

$$
\vec{F}_{v}^{\prime}=e \mu[\vec{v} \times \vec{H}]
$$

or

$$
\vec{E}_{v}^{\prime}=\mu[\vec{v} \times \vec{H}],
$$

and it is final

$$
\vec{F}^{\prime}=e \vec{E}+e \vec{E}_{v}^{\prime}=-e \frac{\partial \vec{A}_{H}}{\partial t}+e \mu[\vec{v} \times \vec{H}] .
$$

Can seem that Eq. (2.11) presents Lorentz force, however, this not thus. In this equation, in contrast to the Lorentz force the field $\vec{E}$ is induction. In order to obtain the total force, which acts on the charge, necessary to the right side Eq. (2.11) to add the term $-e \operatorname{grad} \varphi$

$$
\vec{F}_{\Sigma}^{\prime}=-e \operatorname{grad} \varphi+e \vec{E}+e \mu[\vec{v} \times \vec{H}],
$$

where $\varphi$ is scalar potential at the observation point. In this case Eq. (2.7) can be rewritten as follows:

$\vec{E}^{\prime}=-\mu \frac{\partial \vec{A}_{H}}{\partial t}-\mu(\vec{v} \nabla) \vec{A}_{H}+\mu \operatorname{grad}\left(\vec{v} \vec{A}_{H}\right)-\operatorname{grad} \varphi(2.12)$

or 


$$
\vec{E}^{\prime}=-\mu \frac{d \vec{A}_{H}}{d t}+\operatorname{grad}(\mu(\vec{v} \vec{A})-\varphi) .
$$

If both parts of Eq. (2.12) are multiplied by the magnitude of the charge, then will come out the total force, which acts on the charge. From Lorentz force it will differ in terms of the force $-e \mu \frac{\partial \vec{A}_{H}}{\partial t}$. From Eq. (2.13) it is evident that the value $\mu(\vec{v} \vec{A})-\varphi$ plays the role of the generalized scalar potential. After taking rotor from both parts of Eq. (2.13) and taking into account that rot $\operatorname{grad}=0$, we will obtain

$$
\text { rot } E^{\prime}=-\mu \frac{d \vec{H}}{d t} .
$$

If we in this equation replace total derivative by the quotient, then we will obtain the Maxwell first equation.

After performing this operation, we obtained the Maxwell first equation, but they lost information about power interaction of the moving charge with the magnetic field.

This examination maximally explained the physical picture of mutual induction. We specially looked to this question from another point of view, in order to permit those contradictory judgments, which occur in the fundamental monograph according to the theory of electricity.

Previously Lorentz force was considered as the fundamental experimental postulate, not connected with the law of induction. By calculation to obtain last term of the right side of Eq. (2.11) was only within the framework special relativity (SP), after introducing two postulates of this theory. In this case all terms of Eq. (2.11) are obtained from the law of induction, using the Galileo conversions. Moreover Eq. (2.11) this is a complete law of mutual induction, if it are written down in the terms of vector potential. And this is the very thing rule, which gives possibility, knowing fields in one IRF, to calculate fields in another.

The structure of the forces, which act on the moving charge, is easy to understand based on the example of the case, when the charge moves between two parallel planes, along which flows the current (Figure 1). Let us select for the coordinate axis in such a way that the axis $z$ would be directed normal to planes, and the axis $y$ was parallel.

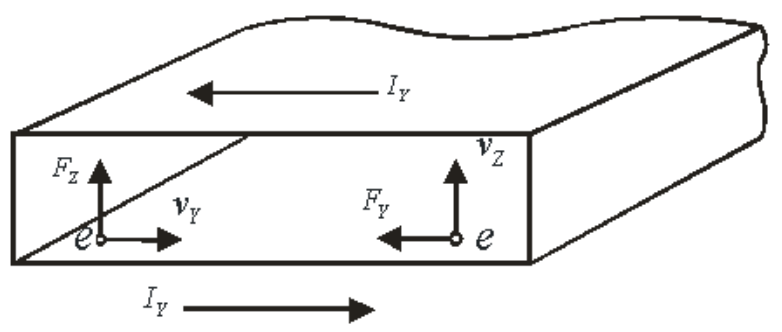

Figure 1. Forces, which act on the charge, which moves in the field of vector potential.

Then the magnetic field $H_{x}$ between them will be equal to the specific current $I_{y}$, which flows along the plates. If the vector potential on the lower plate is equal to zero, then its $A_{y}$ is the component, calculated off the lower plate, will grow according to the law

$$
A_{y}=I_{y} z
$$

If charge moves in the direction of the axis of $y$ near the lower plate with the speed $v_{y}$, then the force $F_{z}$, which acts on the charge, is determined by last term of Eq. (2.6) and it is equal

$$
F_{z}=e \mu v_{y} I_{y}
$$

Is directed this force from the lower plate toward the upper.

If charge moves along the axis of $z$ from the lower plate to the upper with the speed $v_{z}=v_{y}$, then for finding the force should be used already second term of the right side of Eq. (2.6). This force in the absolute value is again equal to the force, determined by Eq. (2.14), and is directed to the side opposite to axis $y$. With any other directions of motion the composite force will be the vector sum of two forces, been last terms of Eq. (2.6). However, the summary amount of this force will be determined by Eq. (2.11), and this force will be always normal to the direction of the motion of charge. Earlier was considered the presence of this force as the action of the Lorentz force, whose nature was obscure, and it was introduced as experimental postulate. It is now understandable that it is the consequence of the combined action of two forces, different in their nature, whose physical sense is now clear.

Understanding the structure of forces gives to us the possibility to look to the already known phenomena from other side. With which is connected existence of the forces, which do extend loop with the current? In this case this circumstance can be interpreted not as the action of Lorentz force, but from an energy point of view. The current, which flows through the element of annular turn is located in the field of the vector potential, created by the remaining elements of this turn, and, therefore, it has it stored up potential energy. The force, which acts on this element, is caused by the presence of the potential gradient energy of this element and is proportional to the gradient to the scalar product of the current strength to the vector potential at the particular point. Thus, it is possible to explain the origin of ponderomotive (mechanical) forces. If current broken into the separate current threads, then they all will separately create the field of vector potential. Summary field will act on each thread individually, and, in accordance with last term of the right side of Eq. (2.6), this will lead to the mutual attraction. Both in the first and in the second case in accordance with the general principles system is approached the minimum of potential energy.

One should emphasize that in Eqs. (2.8) and (2.9) all fields have induction origin, and they are connected first with of the local derivative of vector potential, then by the motion of charge in the three-dimensional changing field of this potential. If fields in the time do not change, then in the right side of Eqs. (2.8) and (2.9) remain only last terms, and they explain the work of all existing electric generators with moving mechanical parts, including the work of unipolar generator. Equation (2.7) gives the possibility to physically explain all composing tensions 
electric fields, which appears in the fixed and that moving the coordinate systems. In the case of unipolar generator in the formation of the force, which acts on the charge, two last addend right sides of equality (2.7) participate, introducing identical contributions.

With conducting of experiments Faraday established that in the outline is induced the current, when in the adjacent outline direct current is switched on or is turned off or adjacent outline with the direct current moves relative to the first outline. Therefore in general form Faraday law is written as follows:

$$
\oint \vec{E}^{\prime} d \vec{l}^{\prime}=-\frac{d \Phi_{B}}{d t} .
$$

This writing of law indicates that with the determination of the circulation of $\vec{E}$ in the moving IRF, near $\vec{E}$ and $d \vec{l}$ must stand primes and should be taken total derivative. But if circulation is determined in the fixed IRF, then primes near $\vec{E}$ and $d \vec{l}$ be absent, but in this case to the right in expression (2.15) must stand particular time derivative.

Complete time derivative in Eq. (2.15) indicates the independence of the eventual result of appearance e.m.f. in the outline from the method of changing the flow. Flow can change both due to the change of $\vec{B}$ with time and because the system, in which is measured the circulation $\oint \vec{E}^{\prime} d \vec{l}^{\prime}$, it moves in the three-dimensional changing field $\vec{B}$. The value of magnetic flux in Eq. (2.15) is determined from the equation

$$
\Phi_{B}=\int \vec{B} d \vec{s}^{\prime}
$$

where the magnetic induction $\vec{B}=\mu \vec{H}$ is determined in the fixed IRF, and the element $d \vec{s}^{\prime}$ is determined in the moving system.

Taking into account Eq. (2.15), from Eq. (2.16) we obtain

$$
\oint \vec{E}^{\prime} d \vec{l}^{\prime}=-\frac{d}{d t} \int \vec{B} d \vec{s}^{\prime}
$$

and further, since $\frac{d}{d t}=\frac{\partial}{\partial t}+\vec{v}$ grad, let us write down [3]

$$
\oint \vec{E}^{\prime} d \vec{l}^{\prime}=-\int \frac{\partial \vec{B}}{\partial t} d \vec{s}^{\prime}-\int[\vec{B} \times \vec{v}] d \overrightarrow{l^{\prime}}-\int \vec{v} \operatorname{div} \vec{B} d \vec{s}^{\prime}
$$

In this case contour integral is taken on the outline $d \vec{l}^{\prime}$, which covers the area of $d \vec{s}^{\prime}$. Let us immediately note that entire following presentation will be conducted under the assumption the validity of the Galileo conversions, i.e., $d \vec{l}^{\prime}=d l$ and $d \vec{s}^{\prime}=d \vec{s}$. From (2.17) follows the known result

$$
\text { of } \vec{E}^{\prime}=\vec{E}+[\vec{v} \times \vec{B}] \text {, }
$$

from which follows that during the motion in the magnetic field the additional electric field, determined by last term of equation appears (2.18). Let us note that this equation is obtained not by the introduction of postulate about the Lorentz force, or from the Lorenz conversions, but directly from the Faraday law, moreover within the framework the conversions of Galileo. Thus, Lorentz force is the direct consequence of the law of magnetoelectric induction.

The equation follows from the Ampere law

$$
\vec{H}=\operatorname{rot} \vec{A}_{H} \text {. }
$$

Then Eq. (2.17) can be rewritten

$$
\vec{E}^{\prime}=-\mu \frac{\partial A_{H}}{\partial t}+\mu[\vec{v} \times \operatorname{rot} \vec{A}]
$$

and further

$$
\vec{E}^{\prime}=-\mu \frac{\partial \vec{A}_{H}}{\partial t}-\mu(\vec{v} \nabla) \vec{A}_{H}+\mu \operatorname{grad}\left(\vec{v} \vec{A}_{H}\right) .
$$

Again came out Eq. (2.7), but it is obtained directly from the Faraday law. True, and this way thus far not shedding light on physical nature of the origin of Lorentz force, since the true physical causes for appearance and magnetic field and vector potential to us nevertheless are not thus far clear.

With the examination of the forces, which act on the charge, we limited to the case, when the time lag, necessary for the passage of signal from the source, which generates vector potential, to the charge itself was considerably less than the period of current variations in the conductors. Now let us remove this limitation.

The Maxwell second equation in the terms of vector potential can be written down as follows:

$$
\operatorname{rot} \operatorname{rot} \vec{A}_{H}=\vec{j}\left(\vec{A}_{H}\right) \text {, }
$$

where $\vec{j}\left(\vec{A}_{H}\right)$ is certain functional from $\vec{A}_{H}$, depending on the properties of the medium in question. If is carried out Ohm law $\vec{j}=\sigma \vec{E}$, then

$$
\vec{j}\left(\vec{A}_{H}\right)=-\sigma \mu \frac{\partial \vec{A}_{H}}{\partial t} .
$$

For the free space takes the form:

$$
\vec{j}\left(\vec{A}_{H}\right)=-\mu \varepsilon \frac{\partial^{2} \vec{A}_{H}}{\partial t^{2}} .
$$

For the free charges the functional takes the form:

$$
\text { of } \vec{j}\left(\vec{A}_{H}\right)=-\frac{\mu}{L_{k}} \vec{A}_{H} \text {, }
$$

where $L_{k}=\frac{m}{n e^{2}}$ is kinetic inductance of charges [4]. In this equation $m$ is the mass of charge, $e$ is the magnitude of the charge, $n$ is charge density.

Equations (2.21 - 2.23) reflect well-known fact about existence of three forms of the electric current: active and two reactive. Each of them has characteristic dependence on the vector potential. This dependence determines the rules of the propagation of vector potential in different media. Here one should emphasize that Eqs. (2.21 - 2.23) assume not only the presence of current, but also the presence of those material media, in which such currents can leak. The conduction current, determined by Eqs. (2.21) and (2.23), can the leak through the conductors, in which there are free current carriers. Bias current, can the 
leak through the free space or the dielectrics. For the free space Eq. (2.20) takes the form:

$$
\operatorname{rot} \operatorname{rot} \vec{A}_{H}=-\mu \varepsilon \frac{\partial^{2} \vec{A}_{H}}{\partial t^{2}} .
$$

This wave equation, which attests to the fact that the vector potential can be extended in the free space in the form of plane waves, and it on its information capability does not be inferior to the wave equations, obtained from Maxwell's equations. This equation on its information capability does not be inferior to wave equations for the electrical and magnetic field on, obtained from Maxwell equations.

Everything said attests to the fact that in the classical electrodynamics the vector potential has important significance. Its use shedding light on many physical phenomena, which previously were not intelligible. And, if it will be possible to explain physical nature of this potential, then is solved the very important problem both of theoretical and applied nature.

\section{Laws of the Electromagnetic Induction}

The Faraday law shows, how a change in the magnetic field on it leads to the appearance of electrical field on. However, does arise the question about that, it does bring a change in the electrical field on to the appearance of magnetic field on? In the case of the absence of conduction currents the the Maxwell second equation appears as follows:

$$
\operatorname{rot} \vec{H}=\varepsilon \frac{\partial \vec{E}}{\partial t}=\frac{\partial \vec{D}}{\partial t},
$$

where $\vec{D}=\varepsilon \vec{E}$ is electrical induction.

And further

$$
\oint \vec{H} d \vec{l}=\frac{\partial \Phi_{E}}{\partial t}
$$

where $\Phi_{E}=\int \vec{D} d \vec{s}$ is the flow of electrical induction.

However for the complete description of the processes of the mutual electrical induction of Eq. (3.1) is insufficient. As in the case Faraday law, should be considered the circumstance that the flow of electrical induction can change not only due to the local derivative of electric field on the time, but also because the outline, along which is produced the integration, it can move in the three-dimensional changing electric field. This means that in Eq. (3.1), as in the case Faraday law, should be replaced the partial derivative by the complete. Designating by the primes of field and circuit elements in moving IRF, we will obtain:

$$
\oint \vec{H}^{\prime} d \vec{l}^{\prime}=\frac{d \Phi_{E}}{d t}
$$

and further

$$
\oint \vec{H}^{\prime} d \vec{l}^{\prime}=\int \frac{\partial \vec{D}}{\partial t} d \vec{s}^{\prime}+\oint[\vec{D} \times \vec{v}] d \vec{l}^{\prime}+\int \vec{v} \operatorname{div} \vec{D} d \vec{s}^{\prime} . \text { (3.2) }
$$

For the electrically neutral medium $\operatorname{div} \vec{E}=0$, therefore the last member of right side in this expression will be absent. For this case Eq. (3.2) will take the form:

$$
\oint \vec{H}^{\prime} d \vec{l}^{\prime}=\int \frac{\partial \vec{D}}{\partial t} d \vec{s}^{\prime}+\oint[\vec{D} \times \vec{v}] d \vec{l}^{\prime}
$$

If we in this equation pass from the contour integration to the integration for the surface, then we will obtain:

$$
\operatorname{rot} \vec{H}^{\prime}=\frac{\partial \vec{D}}{\partial t}+\operatorname{rot}[\vec{D} \times \vec{v}]
$$

If we, based on this equation, write down fields in this inertial system, then prime near $\vec{H}$ and second member of right side will disappear, and we will obtain the bias current, introduced by Maxwell. But Maxwell introduced this parameter, without resorting to the law of electromagnetic induction. If his law of magnetoelectric induction Faraday derived on the basis experiments with the magnetic fields, then experiments on the establishment of the validity of Eq. (3.2) cannot be at that time conducted was, since for conducting this experiment sensitivity of existing at that time meters did not be sufficient.

On from Eq. (3.4) we obtain for the case of constant electrical field:

$$
\vec{H}_{v}^{\prime}=-\varepsilon[\vec{v} \times \vec{E}] .
$$

It is possible to express the electric field through the rotor of electrical vector potential, after assuming

$$
\vec{E}=\operatorname{rot} \vec{A}_{E} \text {. }
$$

Equation (3.4) taking into account Eq. (3.6) will be written down:

$$
\vec{H}^{\prime}=\varepsilon \frac{\partial \vec{A}_{E}}{\partial t}-\varepsilon\left[\vec{v} \times \operatorname{rot} \vec{A}_{E}\right] .
$$

Further it is possible to repeat all those procedures, which has already been conducted with the magnetic vector potential, and to write down the following equations:

$$
\begin{aligned}
\vec{H}^{\prime} & =\varepsilon \frac{\partial \vec{A}_{E}}{\partial t}+\varepsilon(\vec{v} \nabla) \vec{A}_{E}-\varepsilon \operatorname{grad}\left(\vec{v} \vec{A}_{E}\right), \\
\vec{H}^{\prime} & =\varepsilon \frac{\partial \vec{A}_{E}}{\partial t}-\varepsilon\left[\vec{v} \times \operatorname{rot} \vec{A}_{E}\right], \\
\vec{H}^{\prime} & =\varepsilon \frac{d A_{E}}{d t}-\varepsilon \operatorname{grad}\left(\vec{v} A_{E}\right) .
\end{aligned}
$$

Is certain, the study of this problem it would be possible, as in the case the law of magnetoelectric induction, to begin from the introduction of the vector of $\vec{A}_{E}$. This procedure was for the first time proposed in article [5].

The introduction of total derivatives in the laws of induction substantially explains physics of these processes and gives the possibility to isolate the force components, which act on the charge. This method gives also the possibility to obtain transformation laws fields on upon transfer of one IRF to another.

\section{Plurality of the Forms of the Writing of the Electrodynamic Laws}

In the previous paragraph it is shown that the magnetic and electric fields can be expressed through their vector potentials 


$$
\begin{aligned}
\vec{H} & =\operatorname{rot} \vec{A}_{H}, \\
\vec{E} & =\operatorname{rot} \vec{A}_{E} .
\end{aligned}
$$

Consequently, Maxwell equations can be written down with the aid of these potentials:

$$
\begin{aligned}
& \operatorname{rot} \vec{A}_{E}=-\mu \frac{\partial \vec{A}_{H}}{\partial t} \\
& \operatorname{rot} \vec{A}_{H}=\varepsilon \frac{\partial \vec{A}_{E}}{\partial t} .
\end{aligned}
$$

For each of these potentials it is possible to obtain wave equation, in particular

$$
\text { rot rot } \vec{A}_{E}=-\varepsilon \mu \frac{\partial^{2} \vec{A}_{E}}{\partial t^{2}}
$$

and to consider that in the space are extended not the magnetic and electric fields, but the field of electrical vector potential.

In this case, as can easily be seen of the Eqs. (4.1 - 4.4), magnetic and electric field they will be determined through this potential by the equations:

$$
\begin{aligned}
& \vec{H}=\varepsilon \frac{\partial \vec{A}_{E}}{\partial t} \\
& \vec{E}=\operatorname{rot} \vec{A}_{E} .
\end{aligned}
$$

Space derivative rot $\vec{A}_{E}$ and local time derivative $\frac{\partial \vec{A}_{E}}{\partial t}$ are connected with wave equation (4.5).

Thus, the use only of one electrical vector potential makes it possible to completely solve the task about the propagation of electrical and magnetic field on. Taking into account (4.6), Pointing vector can be written down only through the vector $\vec{A}_{E}$ :

$$
\vec{P}=\varepsilon\left[\frac{\partial \vec{A}_{E}}{\partial t} \times \operatorname{rot} \vec{A}_{E}\right] .
$$

Characteristic is the fact that with this method of examination necessary condition is the presence at the particular point of space both the time derivatives, and space derivative of one and the same potential.

This task can be solved by another method, after writing down wave equation for the magnetic vector potential:

$$
\text { rot rot } \vec{A}_{H}=-\varepsilon \mu \frac{\partial^{2} \vec{A}_{H}}{\partial t^{2}} .
$$

In this case magnetic and electric fields will be determined by the equations:

$$
\begin{aligned}
& \vec{H}=\operatorname{rot} \vec{A}_{H} \\
& \vec{E}=-\mu \frac{\partial \vec{A}_{H}}{\partial t} .
\end{aligned}
$$

Pointing vector in this case can be found from the following equation:

$$
\vec{P}=-\mu\left[\frac{\partial \vec{A}_{H}}{\partial t} \times \operatorname{rot} \vec{A}_{H}\right] .
$$

Space derivative rot $\vec{A}_{H}$ and local time derivative of $\frac{\partial \vec{A}_{H}}{\partial t}$ are connected with wave equation (4.7).

But it is possible to enter and differently, after introducing, for example, the electrical and magnetic currents

$$
\begin{aligned}
& \vec{j}_{E}=\operatorname{rot} \vec{H}, \\
& \vec{j}_{H}=\operatorname{rot} \vec{E} .
\end{aligned}
$$

The equations also can be recorded for these currents:

$$
\begin{aligned}
& \text { rot } \vec{j}_{H}=-\mu \frac{\partial \vec{j}_{E}}{\partial t}, \\
& \text { rot } \vec{j}_{E}=\varepsilon \frac{\partial \vec{j}_{H}}{\partial t} .
\end{aligned}
$$

This system in its form and information concluded in it differs in no way from Maxwell equations, and it is possible to consider that in the space the magnetic or electric currents are extended. And the solution of the problem of propagation with the aid of this method will again include complete information about the processes of propagation.

The method of the introduction of new vector examined field on it is possible to extend into both sides ad infinitum, introducing all new vectorial fields. Naturally in this case should be introduced additional calibrations. Thus, there is an infinite set of possible writings of electrodynamic laws, but they all are equivalent according to the information concluded in them. This approach was for the first time demonstrated in the article [5].

\section{Concept of the frequency dispersion of dielectric constant and its physical interpretation}

By all is well known this phenomenon as rainbow. This phenomenon is owing with the dependence on the frequency of the phase speed of the electromagnetic waves, passing through the drops of rain. Since water is dielectric, with the explanation of this phenomenon J. Heaviside R. Wul assumed that this dispersion was connected with the frequency dispersion (dependence on the frequency) of the dielectric constant of water. Since then this point of view is ruling $[6,7,8,9]$.

However very creator of the fundamental equations of electrodynamics Maxwell considered that these parameters on frequency do not depend, but they are fundamental constants. As the idea of the dispersion of dielectric and magnetic constant was born, and what way it was past, sufficiently colorfully characterizes quotation from the monograph of well well-known specialists in the field of physics of plasma [6]: "J. itself. Maxwell with the formulation of the equations of the electrodynamics of material media considered that the dielectric and magnetic constants are the constants (for this reason they long time they were considered as the constants). It is considerably later, already at the beginning of this century with the explanation of the optical dispersion phenomena (in particular the phenomenon of rainbow) of J. Heaviside R. Wul showed that the dielectric and magnetic constants are the functions of frequency. But very recently, in the 
middle of the 50's, physics they came to the conclusion that these values depend not only on frequency, but also on the wave vector. On the essence, this was the radical breaking of the existing ideas. It was how a serious, is characterized the case, which occurred at the seminar 1 . D. Landau into 1954. During the report A. I. Akhiezer on this theme of Landau suddenly exclaimed, after smashing the speaker: "This is delirium, since the refractive index cannot be the function of refractive index". Note that this said l. D. Landau - one of the outstanding physicists of our time”.

It is incomprehensible from the given quotation, that precisely had in the form Landau. However, its subsequent publications speak, that it accepted this concept [7].

Point out, that rights there was Maxwell, who considered that the dielectric and magnetic constant of material media on frequency they do not depend. However, in a number of fundamental monograph on electrodynamics $[6,7,8,9]$ are committed conceptual, systematic and physical errors, as a result of which in physics they penetrated and solidly in it were fastened such metaphysical concepts as the frequency dispersion of the dielectric constant of material media and, in particular, plasma. These physical errors penetrated in all spheres of physics and technology. This systematic and physical error became possible for that reason, that without the proper understanding of physics of the proceeding processes occurred the substitution of physical concepts by mathematical symbols, which appropriated physical, but are more accurate metaphysical, designations, which do not correspond to their physical sense.

\section{Plasmo-like Media}

By plasma media we will understand such, in which the charges can move without the losses. To such media in the first approximation, can be related the superconductors, free electrons or ions in the vacuum. In the media indicated the equation of motion of electron takes the form:

$$
m \frac{d \vec{v}}{d t}=e \vec{E},
$$

where $m$ is mass electron, $e$ is the electron charge, $\vec{E}$ is the tension of electric field, $\vec{v}$ is speed of the motion of charge.

In the article [10] it is shown that this equation can be used also for describing the electron motion in the hot plasma.

Using an expression for the current density

$$
\vec{j}=n e \vec{v},
$$

from (5.1) we obtain the current density of the conductivity

$$
\vec{j}_{L}=\frac{n e^{2}}{m} \int \vec{E} d t .
$$

In Eqs. (5.2) and (5.3) the value $n$ represents electron density. After introducing the designation

$$
L_{k}=\frac{m}{n e^{2}}
$$

we find

$$
\vec{j}_{L}=\frac{1}{L_{k}} \int \vec{E} d t .
$$

In this case the value of $L_{k}$ presents the specific kinetic inductance of charge carriers [4,11,12]. Its existence connected with the fact that charge, having a mass, possesses inertia properties. For the case, when electric field changes according to the law $\vec{E}=\vec{E}_{0} \sin \omega t$, Eq. (5.5) will be written down:

$$
\vec{j}_{L}=-\frac{1}{\omega L_{k}} \vec{E}_{0} \cos \omega t .
$$

For the mathematical description of electrodynamic processes the trigonometric functions will be here and throughout, instead of the complex quantities, used so that would be well visible the phase equations between the vectors, which represent electric fields and current densities.

From Eqs. (5.5) and (5.6) is evident that $\vec{j}_{L}$ presents inductive current, since its phase is late with respect to the tension of electric field to the angle $\frac{\pi}{2}$.

If charges are located in the vacuum, then during the presence of summed current it is necessary to consider bias current

$$
\vec{j}_{\varepsilon}=\varepsilon_{0} \frac{\partial \vec{E}}{\partial t}=\varepsilon_{0} \vec{E}_{0} \cos \omega t .
$$

Is evident that this current bears capacitive nature, since its phase anticipates the phase of the tension of electrical to the angle $\frac{\pi}{2}$. Summary current density will be written down $[5,13,14,15]$ :

$$
\vec{j}_{\Sigma}=\varepsilon_{0} \frac{\partial \vec{E}}{\partial t}+\frac{1}{L_{k}} \int \vec{E} d t,
$$

or

$$
\vec{j}_{\Sigma}=\left(\omega \varepsilon_{0}-\frac{1}{\omega L_{k}}\right) \vec{E}_{0} \cos \omega t .
$$

If electrons are located in the material medium, then should be considered the presence of the positively charged ions. However, the presence of ions usually is not considered, since. their mass is considerably greater than in electrons.

In Eq. (6.7) the value, which stands in the brackets, presents summary susceptance of this medium $\sigma_{\Sigma}$ and it consists it, in turn, of the the capacitive $\sigma_{C}$ and by the inductive $\sigma_{L}$ of the susceptance

$$
\sigma_{\Sigma}=\sigma_{C}+\sigma_{L}=\omega \varepsilon_{0}-\frac{1}{\omega L_{k}} .
$$

Equation (5.7) can be rewritten and differently:

$$
\vec{j}_{\Sigma}=\omega \varepsilon_{0}\left(1-\frac{\omega_{0}^{2}}{\omega^{2}}\right) \vec{E}_{0} \cos \omega t,
$$


where $\omega_{0}=\sqrt{\frac{1}{L_{k} \varepsilon_{0}}}$ is plasma frequency of Langmuir vibrations.

And large temptation here appears to name the value

$$
\varepsilon^{*}(\omega)=\varepsilon_{0}\left(1-\frac{\omega_{0}^{2}}{\omega^{2}}\right)=\varepsilon_{0}-\frac{1}{\omega^{2} L_{k}},
$$

by the depending on the frequency dielectric constant of plasma, that also is made in all existing articles on physics of plasma. But this is incorrect, since this mathematical symbol is the composite parameter, into which simultaneously enters the dielectric constant of vacuum and the specific kinetic inductance of charges.

Let us introduce the determination of the concept of the dielectric constant of medium for the case of variables fields on for the purpose of further concrete definition of the study of the problems of dispersion.

If we examine any medium, including plasma, then current density it will be determined by three components, which depend on the electric field. The current of resistance losses will coincide in the phase with the phase of electric field. The capacitive current, determined by first-order derivative of electric field from the time, will anticipate the tension of electric field on the phase to $\frac{\pi}{2}$. This current is called bias current. The conduction current, connected with the motion of free charges and determined by integral of the electric field from the time, will lag behind the electric field on the phase to $\frac{\pi}{2}$. All three components of current indicated will enter into the Maxwell second equation and others components of currents be it cannot. Moreover all these three components of currents will be present in any nonmagnetic regions, in which there are losses. Therefore it is completely natural, the dielectric constant of any medium to define as the coefficient, confronting that term, which is determined by the derivative of electric field by the time in the Maxwell second equation . In this case one should consider that the dielectric constant cannot be negative value. This connected with the fact that through this parameter is determined energy of electrical field on, which can be only positive.

Without having introduced this clear determination of dielectric constant, Landau begins the examination of the behavior of plasma in the ac fields [7]. In this case it does not extract separately bias current and conduction current, one of which is determined by derivative, but by another integral, but is introduced the united coefficient, which unites these two currents, introducing the dielectric constant of plasma. It makes this error for that reason, that in the case of harmonic oscillations the form of the function, which determine and derivative and integral, is identical, and they are characterized by only sign. Performing this operation, Landau does not understand, that in the case of harmonic electrical field on in the plasma there exist two different currents. One of them is bias current in the vacuum and is determined by derivative of electric field. Another current is conduction current and is determined by integral of the electric field. Moreover these two currents differ in the phase to 180 degrees. But since both currents depend on frequency, between them occurs competition. The conduction current predominates with the low frequencies, the bias current, on the contrary, predominates with the high. However, in the case of the equality of these currents, which occurs at the plasma frequency, occurs current resonance.

Is accurate another point of view. Equation (5.7) can be rewritten and differently:

$$
\vec{j}_{\Sigma}=-\frac{\left(\frac{\omega^{2}}{\omega_{0}^{2}}-1\right)}{\omega L} \vec{E}_{0} \cos \omega t
$$

and to introduce another mathematical symbol

$$
L^{*}(\omega)=\frac{L_{k}}{\left(\frac{\omega^{2}}{\omega_{0}^{2}}-1\right)}=\frac{L_{k}}{\omega^{2} L_{k} \varepsilon_{0}-1} .
$$

In this case also appears temptation to name this bending coefficient on the frequency kinetic inductance. But this value it is not possible to call inductance also, since this also the composite parameter, which includes those not depending on the frequency kinetic inductance and the dielectric constant of vacuum.

Consequently, it is possible to write down:

$$
\vec{j}_{\Sigma}=\omega \varepsilon^{*}(\omega) \vec{E}_{0} \cos \omega t,
$$

or

$$
\vec{j}_{\Sigma}=-\frac{1}{\omega L^{*}(\omega)} \quad \vec{E}_{0} \cos \omega t
$$

But this altogether only the symbolic mathematical record of one and the same Eq. (5.7). Both equations are equivalent. But view neither $\varepsilon^{*}(\omega)$ nor $L^{*}(\omega)$ by dielectric constant or inductance are from a physical point. The physical sense of their names consists of the following:

$$
\varepsilon^{*}(\omega)=\frac{\sigma_{X}}{\omega}
$$

i.e. $\varepsilon^{*}(\omega)$ presents summary susceptance of medium, divided into the frequency, and

$$
L_{k} *(\omega)=\frac{1}{\omega \sigma_{X}}
$$

it represents the reciprocal value of the article of frequency and susceptance of medium.

As it is necessary to enter, if at our disposal are values $\varepsilon^{*}(\omega)$ and $L^{*}(\omega)$, and we should calculate total specific energy? Natural to substitute these values in the formulas, which determine energy of electrical field on

$$
W_{E}=\frac{1}{2} \varepsilon_{0} E_{0}^{2}
$$

and kinetic energy of charge carriers

$$
W_{j}=\frac{1}{2} L_{k} j_{0}^{2}
$$

is cannot simply because these parameters are neither dielectric constant nor inductance. It is not difficult to 
show that in this case the total specific energy can be obtained from the equation

$$
W_{\Sigma}=\frac{1}{2} \cdot \frac{d\left(\omega \varepsilon^{*}(\omega)\right)}{d \omega} E_{0}^{2}
$$

from where we obtain

$$
W_{\Sigma}=\frac{1}{2} \varepsilon_{0} E_{0}^{2}+\frac{1}{2} \frac{1}{\omega^{2} L_{k}} E_{0}^{2}=\frac{1}{2} \varepsilon_{0} E_{0}^{2}+\frac{1}{2} L_{k} j_{0}^{2} .
$$

We will obtain the same result, after using the formula

$$
W=\frac{1}{2} \frac{d\left[\frac{1}{\omega L_{k} *(\omega)}\right]}{d \omega} E_{0}^{2} .
$$

The given equations show that the specific energy consists of potential energy of electrical field on and to kinetic energy of charge carriers.

With the examination of any media by our final task appears the presence of wave equation. In this case this problem is already practically solved. Maxwell equations for this case take the form:

$$
\begin{aligned}
& \operatorname{rot} \vec{E}=-\mu_{0} \frac{\partial \vec{H}}{\partial t}, \\
& \operatorname{rot} \vec{H}=\varepsilon_{0} \frac{\partial \vec{E}}{\partial t}+\frac{1}{L_{k}} \int \vec{E} d t,
\end{aligned}
$$

where $\varepsilon_{0}$ and $\mu_{0}$ is dielectric and magnetic constant of vacuum.

System of equations (5.10) completely describes all properties of the conductors, in which be absent the ohmic losses. From (5.10) we obtain

$$
\text { rot rot } \vec{H}+\mu_{0} \varepsilon_{0} \frac{\partial^{2} \vec{H}}{\partial t^{2}}+\frac{\mu_{0}}{L_{k}} \vec{H}=0 .
$$

For the case field on, time-independent, equation (5.11) passes into the equation of London

$$
\text { rot rot } \vec{H}+\frac{\mu_{0}}{L_{k}} \vec{H}=0 \text {, }
$$

where $\lambda_{L}^{2}=\frac{L_{k}}{\mu_{0}}$ is London depth of penetration.

Thus, it is possible to conclude that the equations of London being a special case of equation (5.11), and do not consider bias currents on the superconductor. Therefore they do not give the possibility to obtain the wave equations, which describe the processes of the propagation of electromagnetic waves in the superconductors.

Field on wave equation in this case it appears as follows for the electrical:

$$
\text { rot rot } \vec{E}+\mu_{0} \varepsilon_{0} \frac{\partial^{2} \vec{E}}{\partial t^{2}}+\frac{\mu_{0}}{L_{k}} \vec{E}=0
$$

For constant electrical field on it is possible to write down:

$$
\text { rot rot } \vec{E}+\frac{\mu_{0}}{L_{k}} \vec{E}=0
$$

Consequently, dc fields penetrate the superconductor in the same manner as for magnetic, diminishing exponentially. However, the density of current in this case grows according to the linear law

$$
\vec{j}_{L}=\frac{1}{L_{k}} \int \vec{E} d t .
$$

The carried out examination showed that the dielectric constant of this medium was equal to the dielectric constant of vacuum and this permeability on frequency does not depend. The accumulation of potential energy is obliged to this parameter. Furthermore, this medium is characterized still and the kinetic inductance of charge carriers and this parameter determines the kinetic energy, accumulated on Wednesday.

Consequently, are acquired all necessary data, which characterize the process of the propagation of electromagnetic waves in the plasmo-like media. However, in contrast to the conventional procedure $[7,8,9]$ with this examination nowhere was introduced polarization vector, but as the basis of examination assumed equation of motion and in this case in the Maxwell second equation are extracted all components of current densities explicitly. In this case in the Maxwell second equation are extracted all components of current densities explicitly.

In radio engineering exists the simple method of the idea of radio-technical elements with the aid of the equivalent diagrams. This method is very visual and gives the possibility to present in the form such diagrams elements both with that concentrated and with the distributed parameters. The use of this method will make it possible better to understand, why were committed such significant physical errors during the introduction of the concept of that depending on the frequency dielectric constant.

In order to show that the single volume of conductor or plasma according to its electrodynamic characteristics is equivalent to parallel resonant circuit with the lumped parameters, let us examine parallel resonant circuit. The connection between the voltage $U$, applied to the resonant circuit, and the summed current $I_{\Sigma}$, which flows through this circuit, takes the form

$$
I_{\Sigma}=I_{C}+I_{L}=C \frac{d U}{d t}+\frac{1}{L} \int U d t
$$

where $I_{C}=C \frac{d U}{d t}$ is current, which flows through the capacity, and $I_{L}=\frac{1}{L} \int U d t$ is current, which flows through the inductance.

For the case of the harmonic voltage $U=U_{0} \sin \omega t$ we obtain

$$
I_{\Sigma}=\left(\omega C-\frac{1}{\omega L}\right) U_{0} \cos \omega t
$$

The value, which stands in the brackets, presents summary susceptance $\sigma_{\Sigma}$ of the circuit examined and consists. It consists of the capacitive $\sigma_{C}$ and by the inductive $\sigma_{L}$ of the susceptance 


$$
\sigma_{\Sigma}=\sigma_{C}+\sigma_{L}=\omega C-\frac{1}{\omega L}
$$

In this case Eq. (5.12) can be rewritten as follows:

$$
I_{\Sigma}=\omega C\left(1-\frac{\omega_{0}^{2}}{\omega^{2}}\right) U_{0} \cos \omega t,
$$

where $\omega_{0}^{2}=\frac{1}{L C}$ is the resonance frequency of parallel circuit.

And here, just as in the case of conductors, appears temptation, to name the value

$$
C^{*}(\omega)=C\left(1-\frac{\omega_{0}^{2}}{\omega^{2}}\right)=C-\frac{1}{\omega^{2} L}
$$

by the depending on the frequency capacity. Conducting this symbol it is permissible from a mathematical point of view, however, inadmissible is awarding to it the proposed name, since. This parameter of no relation to the true capacity has and includes in itself simultaneously and capacity and the inductance of outline, which do not depend on frequency. It includes in itself simultaneously and capacity and the inductance of outline, which do not depend on frequency.

Is accurate another point of view. Equation (5.12) can be rewritten and differently:

$$
I_{\Sigma}=-\frac{\left(\frac{\omega^{2}}{\omega_{0}^{2}}-1\right)}{\omega L} U_{0} \cos \omega t,
$$

and to consider that the chain in question not at all has capacities, and consists only of the inductance depending on the frequency

$$
L *(\omega)=\frac{L}{\left(\frac{\omega^{2}}{\omega_{0}^{2}}-1\right)}=\frac{L}{\omega^{2} L C-1}
$$

Using expressions (5.13) and (5.14), let us write down:

$$
I_{\Sigma}=\omega C^{*}(\omega) U_{0} \cos \omega t
$$

or

$$
I_{\Sigma}=-\frac{1}{\omega L^{*}(\omega)} U_{0} \cos \omega t
$$

Equations (5.15) and (5.16) are equivalent, and separately mathematically completely is characterized the chain examined. But view neither $C^{*}(\omega)$ nor $L^{*}(\omega)$ by capacity and inductance are from a physical point, although they have the same dimensionality. The physical sense of their names consists of the following:

$$
C^{*}(\omega)=\frac{\sigma_{X}}{\omega},
$$

i.e. $C^{*}(\omega)$ presents the relation of susceptance of this chain and frequency, and

$$
L^{*}(\omega)=\frac{1}{\omega \sigma_{X}},
$$

it is the reciprocal value of the article of product susceptance and frequency.

Accumulated in the capacity and the inductance energy, is determined from the equations

$$
\begin{gathered}
W_{C}=\frac{1}{2} C U_{0}{ }^{2}, \\
W_{L}=\frac{1}{2} L I_{0}{ }^{2} .
\end{gathered}
$$

how one should enter for enumerating the energy, which was accumulated in the outline, if at our disposal are $C^{*}(\omega)$ and $L^{*}(\omega)$ ? Certainly, to put these equations in equations (5.17) and (5.18) is impossible already at least because these values can be both the positive and negative, and energy in this case is positive value. However, it is not difficult to show that the summary energy, accumulated in the outline, is determined by the expressions:

$$
W_{\Sigma}=\frac{1}{2} \frac{d \sigma_{X}}{d \omega} U_{0}^{2}
$$

or

$$
W_{\Sigma}=\frac{1}{2} \frac{d\left[\omega C^{*}(\omega)\right]}{d \omega} U_{0}^{2},
$$

or

$$
W_{\Sigma}=\frac{1}{2} \frac{d\left(\frac{1}{\omega L^{*}(\omega)}\right)}{d \omega} U_{0}^{2} .
$$

If we paint equations (5.19) or (5.20) and (5.21), then we will obtain identical result, namely:

$$
W_{\Sigma}=\frac{1}{2} C U_{0}^{2}+\frac{1}{2} L I_{0}^{2},
$$

where $U_{0}$ is amplitude of voltage on the capacity, and $I_{0}$ is amplitude of the current, which flows through the inductance.

If we compare the equations, obtained for the parallel resonant circuit and for the conductors, then it is possible to see that they are identical, if we make $E_{0} \rightarrow U_{0}$, $j_{0} \rightarrow I_{0}, \varepsilon_{0} \rightarrow C$ and $L_{k} \rightarrow L$. Thus, the single volume of conductor, with the uniform distribution of electrical field on and current densities in it, it is equivalent to parallel resonant circuit with the lumped parameters indicated. In this case the capacity of this outline is numerically equal to the dielectric constant of vacuum, and inductance is equal to the specific kinetic inductance of charges.

Thus, are obtained all necessary given, which characterize the process of the propagation of electromagnetic waves in the media examined, and it is also shown that in the quasi-static regime the electrodynamic processes in the conductors are similar to processes in the parallel resonant circuit with the lumped parameters. However, in contrast to the conventional procedure $[7,8,9]$ with this examination nowhere was introduced polarization vector, but as the basis of examination assumed equation of motion and in this case in the Maxwell second equation are extracted all components of current densities explicitly. 
Based on the example of monograph [7] let us examine a question about how similar problems, when the concept of polarization vector is introduced are solved for their solution. Paragraph 59 of this monograph, where this question is examined, it begins with the words: "We pass now to the study of the most important question about the rapidly changing electric fields, whose frequencies are unconfined by the condition of smallness in comparison with the frequencies, characteristic for establishing the electrical and magnetic polarization of substance”.

These words mean that that region of the frequencies, where, in connection with the presence of the inertia properties of charge carriers, the polarization of substance will not reach its static values, is examined. With the further consideration of a question is done the conclusion that "in any variable field, including with the presence of dispersion, the polarization vector $\vec{P}=\vec{D}-\varepsilon_{0} \vec{E}$ (here and throughout all formulas cited they are written in the system SI) preserves its physical sense of the electric moment of the unit volume of substance”.

Let us give the still one quotation: "It proves to be possible to establish (unimportantly - metals or dielectrics) maximum form of the function of $\varepsilon(\omega)$ with the high frequencies valid for any bodies. Specifically, the field frequency must be great in comparison with "the frequencies” of the motion of all (or, at least, majority) electrons in the atoms of this substance. With the observance of this condition it is possible with the calculation of the polarization of substance to consider electrons as free, disregarding their interaction with each other and with the atomic nuclei"

Further is written the equation of motion of free electron in the ac field

$$
m \frac{d \vec{v}}{d t}=e \vec{E}
$$

from where its displacement is located

$$
\vec{r}=-\frac{e \vec{E}}{m \omega^{2}} .
$$

Then is indicated that the polarization $\vec{P}$ is a dipole moment of unit volume and the obtained displacement is put into the polarization

$$
\vec{P}=n e \vec{r}=\frac{n e^{2} \vec{E}}{m \omega^{2}} .
$$

In this case point charge is examined, and this operation indicates the introduction of electrical dipole moment for two point charges with the opposite signs, located at a distance $\vec{r}$

$$
\vec{P}_{e}=-e \vec{r} .
$$

This step causes bewilderment, since the point electron is examined, and in order to speak about the electrical dipole moment, it is necessary to have in this medium for each electron another charge of opposite sign, referred from it to the distance $\vec{r}$. In this case is examined the gas of free electrons, in which there are no charges of opposite signs. Further follows the standard procedure, when introduced thus illegal polarization vector is introduced into the dielectric constant

$$
\vec{D}=\varepsilon_{0} \vec{E}+\vec{P}=\varepsilon_{0} \vec{E}-\frac{n e^{2} \vec{E}}{m \omega^{2}}=\varepsilon_{0}\left(1-\frac{1}{\varepsilon_{0} L_{k} \omega^{2}}\right) \vec{E},
$$

and since plasma frequency is determined by the equation

$$
\omega_{p}^{2}=\frac{1}{\varepsilon_{0} L_{k}},
$$

the vector of the induction immediately is written

$$
\vec{D}=\varepsilon_{0}\left(1-\frac{\omega_{p}^{2}}{\omega^{2}}\right) \vec{E}
$$

With this approach it turns out that constant of proportionality

$$
\varepsilon(\omega)=\varepsilon_{0}\left(1-\frac{\omega_{p}^{2}}{\omega^{2}}\right)
$$

between the electric field and the electrical induction, illegally named dielectric constant, depends on frequency.

Precisely this approach led to the fact that all began to consider that the value, which stands in this equation before the vector of electric field, is the dielectric constant depending on the frequency, and electrical induction also depends on frequency. And this it is discussed in all, without the exception, fundamental monograph on the electrodynamics of material media [7,8,9].

But, as it was shown above this parameter it is not dielectric constant, but presents summary susceptance of medium, divided into the frequency. Thus, traditional approach to the solution of this problem from a physical point of view is erroneous. But from a mathematical point of view this approach let us assume however in this case there is no possibility of the calculation of initial conditions with the calculation of integral in the equations, which determine conduction current.

Further into $\S 61$ of article [7] is examined a question about the energy of electrical and magnetic field in the media, which possess by the so-called dispersion. In this case is done the conclusion that equation for the energy of such field on

$$
W=\frac{1}{2}\left(\varepsilon E_{0}^{2}+\mu H_{0}^{2}\right),
$$

of that making precise thermodynamic sense in the usual media, with the presence of dispersion so interpreted be cannot. These words mean that the knowledge of real electrical and magnetic field on with the dispersion insufficiently for determining the difference in the internal energy per unit of volume of substance in the presence field on in their absence. After such statements is given the formula, which gives correct result for enumerating the specific energy of electrical and magnetic field on when the dispersion present,

$$
W=\frac{1}{2} \frac{d(\omega \varepsilon(\omega))}{d \omega} E_{0}^{2}+\frac{1}{2} \frac{d(\omega \mu(\omega))}{d \omega} H_{0}^{2} .
$$

But if we compare the first part of the expression in the right side of Eq. (5.23) with Eq. (5.9), then it is evident that they coincide. This means that in Eq. (5.23) this term presents the total energy, which includes not only 
potential energy of electrical field on, but also kinetic energy of the moving charges.

Therefore conclusion about the impossibility of the interpretation of formula (5.22), as the internal energy of electrical and magnetic field on in the media with the dispersion it is correct. However, this circumstance consists not in the fact that this interpretation in such media is generally impossible. It consists in the fact that for the definition of the value of energy as the thermodynamic parameter is necessary to correctly calculate this energy. In this case it follows taking into account not only electric field, which accumulates potential energy, but also current of the conduction electrons, which accumulate the kinetic kinetic energy of charges (5.8). The conclusion, which now can be made, consists of the following. The conclusion, which now can be made, consists in the fact that, introducing into the custom some mathematical symbols, without understanding of their true physical sense, and, all the more, the awarding to these symbols of physical designations unusual to them, it is possible in the final analysis to lead to the significant errors, that also occurred in the article [7].

\section{Transverse Plasma Resonance}

Is known that the plasma resonance is longitudinal. But the charges, which are varied lengthwise, not to emit transverse radio waves. However, with the explosions of nuclear charges, as a result of which is formed very hot plasma, occurs electromagnetic radiation in the very wide frequency band, up to the long-wave radio-frequency band. Today are not known those of the physical mechanisms, which could explain the appearance of this emission. There were no other resonances of any kind, except plasma, earlier known on existence in the nonmagnetic plasma. But it occurs that in the confined plasma can exist the transverse plasma resonance, whose frequency coincides with the frequency of longitudinal plasma resonance. Specifically, this resonance can be the reason for the emission of electromagnetic waves with the explosions of nuclear charges [13,14,15,17]. For explaining the conditions for the excitation of this resonance let us examine the long line, which consists of two ideally conducting planes, as shown in Figure 2

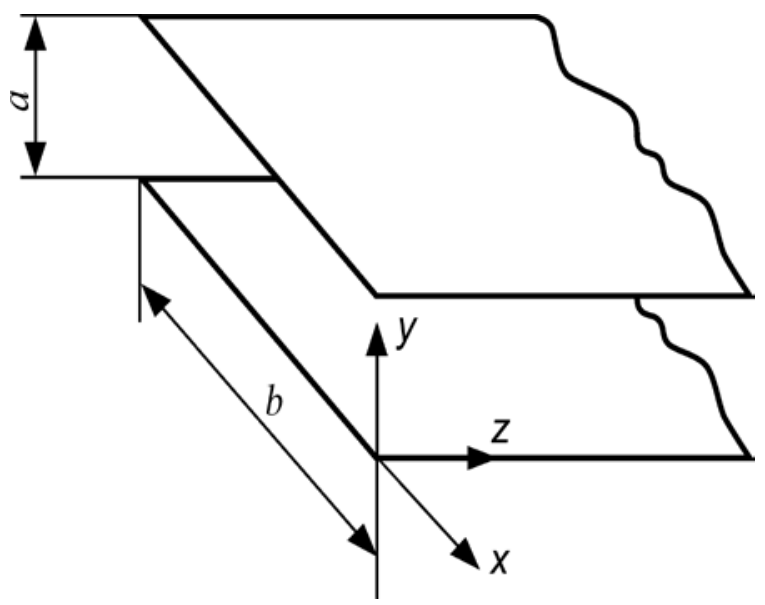

Figure 2. The two-wire circuit, which consists of two ideally conducting planes
Linear capacity and inductance of this line without taking into account edge effects they are determined by the equations:

$$
C_{0}=\varepsilon_{0} \frac{b}{a}, L_{0}=\mu_{0} \frac{a}{b} .
$$

Therefore with an increase in the length of line its total capacitance $C_{\Sigma}=\varepsilon_{0} \frac{b}{a} z$ and summary inductance $L_{\Sigma}=\mu_{0} \frac{a}{b} z$ increase proportional to its length.

If we into the extended line place the plasma, charge carriers in which can move without the losses, and in the transverse direction pass through the plasma the current $I$, then charges, moving with the definite speed, will accumulate kinetic energy. Let us note that here are not examined technical questions, as and it is possible confined plasma between the planes of line how. In this case only fundamental questions, which are concerned transverse plasma resonance in the nonmagnetic plasma, are examined.

Since the transverse current density in this line is determined by the equation

$$
j=\frac{I}{b z}=n e v,
$$

that summary kinetic energy of the moving charges can be written down

$$
W_{k \Sigma}=\frac{1}{2} \frac{m}{n e^{2}} \quad a b z j^{2}=\frac{1}{2} \frac{m}{n e^{2}} \frac{a}{b z} I^{2} .
$$

Equation (6.1) connects the kinetic energy, accumulated in the line, with the square of current; therefore the coefficient, which stands in the right side of this equation before the square of current, is the summary kinetic inductance of line

$$
L_{k \Sigma}=\frac{m}{n e^{2}} \cdot \frac{a}{b z} .
$$

Thus, the value

$$
L_{k}=\frac{m}{n e^{2}}
$$

presents the specific kinetic inductance of charges. Equation (6.3) is obtained for the case of the direct current, when current distribution is uniform.

Subsequently for the larger clarity of the obtained results, together with their mathematical idea, we will use the method of equivalent diagrams. The section the lines examined, long $d z$ can be represented in the form the equivalent diagram, shown in Figure 3 a.

From (6.2) we see that the magnitude $L_{k \Sigma}$ of the growth $z$ not increases, but it decreases. Connected this with the fact that with an increase in $z$ a quantity of parallel-connected inductive elements grows.

The equivalent the schematic of the section of the line, filled with nondissipative plasma, it is shown in Figure 3 б. Line itself in this case will be equivalent to parallel circuit with the lumped parameters:

$$
C=\frac{\varepsilon_{0} b z}{a}, L=\frac{L_{k} a}{b z},
$$


in series with which is connected the inductance

$$
\mu_{0} \frac{a d z}{b} \text {. }
$$
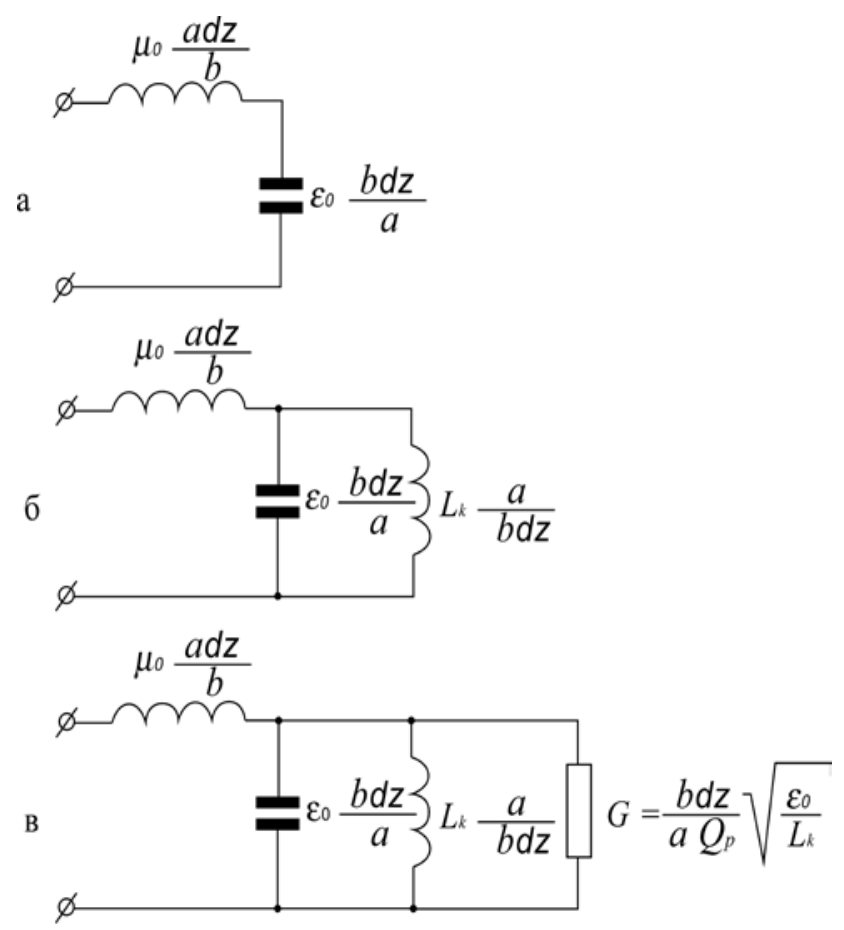

Figure 3. a - the equivalent the schematic of the section of the two-wire circuit; $\sigma$ - the equivalent the schematic of the section of the two-wire circuit, filled with nondissipative plasma; в - the equivalent the schematic of the section of the two-wire circuit, filled with dissipative plasma

But if we calculate the resonance frequency of this outline, then it will seem that this frequency generally not on what sizes depends, actually:

$$
\omega_{\rho}^{2}=\frac{1}{C L}=\frac{1}{\varepsilon_{0} L_{k}}=\frac{n e^{2}}{\varepsilon_{0} m} .
$$

Is obtained the interesting result, which speaks, that the resonance frequency macroscopic of the resonator examined does not depend on its sizes. Impression can be created, that this is plasma resonance, since the obtained value of resonance frequency exactly corresponds to the value of this resonance. But it is known that the plasma resonance characterizes longitudinal waves in the long line they, while occur transverse waves. In the case examined the value of the phase speed in the direction of $z$ is equal to infinity and the wave vector $\vec{k}=0$.

This result corresponds to the solution of system of equations (5.10) for the line with the assigned configuration. In this case the wave number is determined by the equation:

$$
k_{z}^{2}=\frac{\omega^{2}}{c^{2}}\left(1-\frac{\omega_{\rho}^{2}}{\omega^{2}}\right)
$$

and the group and phase speeds

$$
v_{g}^{2}=c^{2}\left(1-\frac{\omega_{\rho}^{2}}{\omega^{2}}\right)
$$

$$
v_{F}^{2}=\frac{c^{2}}{\left(1-\frac{\omega_{\rho}^{2}}{\omega^{2}}\right)}
$$

where $c=\left(\frac{1}{\mu_{0} \varepsilon_{0}}\right)^{1 / 2}$ is speed of light in the vacuum.

For the present instance the phase speed of electromagnetic wave is equal to infinity, which corresponds to transverse resonance at the plasma frequency. Consequently, at each moment of time field on distribution and currents in this line uniform and it does not depend on the coordinate $z$, but current in the planes of line in the direction of is absent. This, from one side, it means that the inductance $L_{\Sigma}$ will not have effects on electrodynamic processes in this line, but instead of the conducting planes can be used any planes or devices, which limit plasma on top and from below.

From equations (6.4), (6.5) and (6.6) is evident that at the point $\omega=\omega_{p}$ occurs the transverse resonance with the infinite quality. With the presence of losses in the resonator will occur the damping, and in the long line in this case of , and in the line will be extended the damped transverse wave, the direction of propagation of which will be normal to the direction of the motion of charges. It should be noted that the fact of existence of this resonance previously was not realized and in the literature it was not described.

\section{Symmetrization of Maxwell Equations and Kinetic Capacity}

If we consider all components of current density in the conductor, then the Maxwell second equation can be written down $[5,13,14,15,17]$ :

$$
\operatorname{rot} \vec{H}=\sigma_{E} \vec{E}+\varepsilon \frac{\partial \vec{E}}{\partial t}+\frac{1}{L_{k}} \int \vec{E} d t
$$

where $\sigma_{E}$ is conductivity of metal.

At the same time, the Maxwell first equation can be written down as follows:

$$
\operatorname{rot} \vec{E}=-\mu \frac{\partial \vec{H}}{\partial t}
$$

where $\mu$ is magnetic permeability of medium. It is evident that equations (7.1) and (7.2) are asymmetrical.

To somewhat improve the symmetry of these equations are possible, introducing into equation (7.2) term linear for the magnetic field, that considers heat losses in the magnetic materials in the variable fields:

$$
\operatorname{rot} \vec{E}=-\sigma_{H} \vec{H}-\mu \frac{\partial \vec{H}}{\partial t}
$$

where $\sigma_{H}$ is conductivity of magnetic currents. But here there is no integral of such type, which is located in the right side of equation (7.1), in this equation. At the same time to us it is known that the atom, which possesses the magnetic moment of $\vec{m}$, placed into the magnetic field, 
and which accomplishes in it precessional motion, has potential energy of $U_{m}=-\mu \vec{m} \vec{H}$. Therefore potential energy can be accumulated not only in the electric fields, but also in the precessional motion of magnetic moments, which does not possess inertia. Similar case is located also in the mechanics, when the gyroscope, which precesses in the field of external gravitational forces, accumulates potential energy. Regarding mechanical precessional motion is also noninertial and immediately ceases after the removal of external forces. For example, if we from under the precessing gyroscope, which revolves in the field of the earth's gravity, rapidly remove support, thus it will begin to fall, preserving in the space the direction of its axis, which was at the moment, when support was removed. The same situation occurs also for the case of the precessing magnetic moment. Its precession is noninertial and ceases at the moment of removing the magnetic field.

Therefore it is possible to expect that with the description of the precessional motion of magnetic moment in the external magnetic field in the right side of Eq. (7.3) can appear a term of the same type as in Eq. (7.1). It will only stand $L_{k}$, i.e., instead $C_{k}$ the kinetic capacity, which characterizes that potential energy, which has the precessing magnetic moment in the magnetic field:

$$
\operatorname{rot} \vec{E}=-\sigma_{H} \vec{H}-\mu \frac{\partial \vec{H}}{\partial t}-\frac{1}{C_{k}} \int \vec{H} d t .
$$

For the first time this idea of the Maxwell first equation taking into account kinetic capacity was given in the articles $[5,18,19]$.

Resonance processes in the plasma and the dielectrics are characterized by the fact that in the process of fluctuations occurs the alternating conversion of electrostatic energy into the kinetic energy of charges and vice versa. This process can be named electrokinetic and all devices: lasers, masers, filters, etc, which use this process, can be named electrokinetic devices. At the same time there is another type of resonance - magnetic. If we use ourselves the existing ideas about the dependence of magnetic permeability on the frequency, then it is not difficult to show that this dependence is connected with the presence of magnetic resonance. In order to show this, let us examine the concrete example of ferromagnetic resonance. If we magnetize ferrite, after applying the stationary field $H_{0}$ in parallel to the axis $z$, the like to relation to the external variable field medium will come out as anisotropic magnetic material with the complex permeability in the form of tensor [20]

$$
\mu=\left(\begin{array}{lcl}
\mu_{T}^{*}(\omega) & -i \alpha & 0 \\
i \alpha & \mu_{T}^{*}(\omega) & 0 \\
0 & 0 & \mu_{L}
\end{array}\right),
$$

where

$$
\mu_{T}^{*}(\omega)=1-\frac{\Omega|\gamma| M_{0}}{\mu_{0}\left(\omega^{2}-\Omega^{2}\right)}, \quad \alpha=\frac{\omega|\gamma| M_{0}}{\mu_{0}\left(\omega^{2}-\Omega^{2}\right)}, \quad \mu_{L}=1,
$$

moreover

$$
\Omega=|\gamma| H_{0}
$$

is natural frequency of precession.

$$
M_{0}=\mu_{0}(\mu-1) H_{0}
$$

is a magnetization of medium. Taking into account (7.4) and (7.5), it is possible to write down

$$
\mu_{T} *(\omega)=1-\frac{\Omega^{2}(\mu-1)}{\omega^{2}-\Omega^{2}} .
$$

Therefore that magnetic permeability of magnetic material depends on frequency, and can arise suspicions, that, as in the case with the plasma, here is some misunderstanding.

If we consider that the electromagnetic wave is propagated along the axis $x$ and there are components fields $H_{y}$ and $H_{z}$, then in this case the Maxwell first equation will be written down:

$$
\operatorname{rot} \vec{E}=\frac{\partial \vec{E}_{Z}}{\partial x}=\mu_{0} \mu_{T} \frac{\partial \vec{H}_{y}}{\partial t} \text {. }
$$

Taking into account (7.6), let us write down

$$
\text { rot } \vec{E}=\mu_{0}\left[1-\frac{\Omega^{2}(\mu-1)}{\omega^{2}-\Omega^{2}}\right] \frac{\partial \vec{H}_{y}}{\partial t} \text {. }
$$

For the case $\omega>>\Omega$ we have

$$
\operatorname{rot} \vec{E}=\mu_{0}\left[1-\frac{\Omega^{2}(\mu-1)}{\omega^{2}}\right] \frac{\partial \vec{H}_{y}}{\partial t} \text {. }
$$

Assuming $H_{y}=H_{y 0} \sin \omega t$ and taking into account that in this case

$$
\frac{\partial \vec{H}_{y}}{\partial t}=-\omega^{2} \int \vec{H}_{y} d t
$$

we will obtain from (7.7)

$$
\operatorname{rot} \vec{E}=\mu_{0} \frac{\partial \vec{H}_{y}}{\partial t}+\mu_{0} \Omega^{2}(\mu-1) \int \vec{H}_{y} d t
$$

or

$$
\operatorname{rot} \vec{E}=\mu_{0} \frac{\partial \vec{H}_{y}}{\partial t}+\frac{1}{C_{k}} \int \vec{H}_{y} d t .
$$

For the case $\omega<<\Omega$ we find

$$
\text { rot } \vec{E}=\mu_{0} \mu \frac{\partial \vec{H}_{y}}{\partial t} \text {. }
$$

Value

$$
C_{k}=\frac{1}{\mu_{0} \Omega^{2}(\mu-1)},
$$

which is introduced in Eq. (7.8), let us name kinetic capacity.

With which is connected existence of this parameter, and its what physical sense? If the direction of magnetic moment does not coincide with the direction of external magnetic field, then the vector of this moment begins to precess around the vector of magnetic field with the 
frequency of $\Omega$. The magnetic moment $\vec{m}$ possesses in this case potential energy $U_{m}=-\vec{m} \cdot \vec{B}$. This energy similar to energy of the charged capacitor is potential, because precessional motion, although is mechanical, however, it not inertia and instantly it does cease during the removal of magnetic field. However, with the presence of magnetic field precessional motion continues until the accumulated potential energy is spent, and the vector of magnetic moment will not become parallel to the vector of magnetic field.

The equivalent diagram of the case examined is given in Figure 4. At the point $\omega=\Omega$ occurs magnetic resonance, in this case $\mu_{T}^{*}(\omega) \rightarrow-\infty$. The resonance frequency of macroscopic magnetic resonator, as can easily be seen of the equivalent diagram, also does not depend on the dimensions of line and is equal $\Omega$. Thus, the parameter

$$
\mu_{H} *(\omega)=\mu_{0}\left[1-\frac{\Omega^{2}(\mu-1)}{\omega^{2}-\Omega^{2}}\right]
$$

is not the frequency dependent magnetic permeability, but it is the combined parameter, including $\mu_{0}, \mu$ and $C_{k}$, which are included on in accordance with the equivalent diagram, depicted in Figure 4.

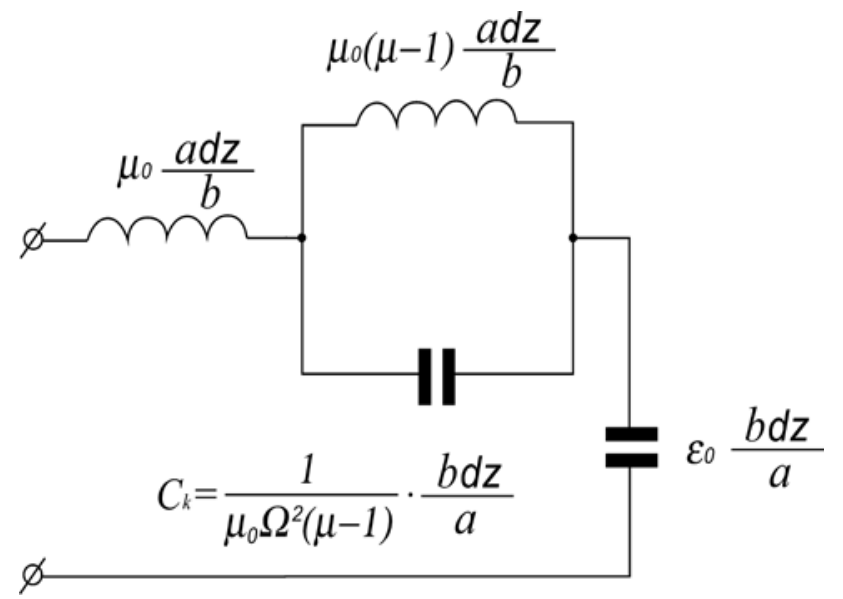

Figure 4. Equivalent the schematic of the two-wire circuit of that filled with magnetic material.

Is not difficult to show that in this case there are three waves: electrical, magnetic and the wave, which carries potential energy, which is connected with the precession of magnetic moments around the vector $H_{0}$. For this reason such waves can be named elektromagneticpotential waves.

\section{Dielectrics}

In the existing literature there are no indications that the kinetic inductance of charge carriers plays some role in the electrodynamic processes in the dielectrics. This not thus. This parameter in the electrodynamics of dielectrics plays not less important role, than in the electrodynamics of conductors. Let us examine the simplest case, when oscillating processes in atoms or molecules of dielectric obey the law of mechanical oscillator [18]. Let us write down the equation of motion of the electromechanical oscillator of

$$
\left(\frac{\beta}{m}-\omega^{2}\right) \vec{r}_{m}=\frac{e}{m} \vec{E}
$$

where $\vec{r}_{m}$ is deviation of charges from the position of equilibrium, $\beta$ is coefficient of elasticity, which characterizes the elastic electrical binding forces of charges in the atoms and the molecules. Introducing the resonance frequency of the bound charges

$$
\omega_{0}=\frac{\beta}{m},
$$

we will obtain from (8.1)

$$
r_{m}=-\frac{e E}{m\left(\omega^{2}-\omega_{o}^{2}\right)} .
$$

Is evident that in Eq. (8.2) as the parameter is present the natural vibration frequency, into which enters the mass of charge. This speaks, that the inertia properties of the being varied charges will influence oscillating processes in the atoms and the molecules.

Since the complete current density on the medium consists of the bias current and conduction current

$$
\operatorname{rot} \vec{H}=\vec{j}_{\Sigma}=\varepsilon_{0} \frac{\partial \vec{E}}{\partial t}+n e \vec{v},
$$

the speed of charge carriers is derivative on the coordinate of their displacement

$$
\vec{v}=\frac{\partial r_{m}}{\partial t}=-\frac{e}{m\left(\omega^{2}-\omega_{o}^{2}\right)} \frac{\partial \vec{E}}{\partial t},
$$

from Eq. (8.2) we find

$$
\operatorname{rot} \vec{H}=\vec{j}_{\Sigma}=\varepsilon_{0} \frac{\partial \vec{E}}{\partial t}-\frac{1}{L_{k d}\left(\omega^{2}-\omega_{0}^{2}\right)} \frac{\partial \vec{E}}{\partial t} .
$$

Let us note that the value

$$
L_{k d}=\frac{m}{n e^{2}}
$$

presents the kinetic inductance of the charges, entering the constitution of atom or molecules of dielectrics, when to consider charges free. Therefore Eq. (8.3) it is possible to rewrite

$$
\operatorname{rot} \vec{H}=\vec{j}_{\Sigma}=\varepsilon_{0}\left(1-\frac{1}{\varepsilon_{0} L_{k d}\left(\omega^{2}-\omega_{0}^{2}\right)}\right) \frac{\partial \vec{E}}{\partial t} .
$$

Since the value

$$
\frac{1}{\varepsilon_{0} L_{k d}}=\omega_{p d}{ }^{2}
$$

it represents the plasma frequency of charges in atoms and molecules of dielectric, if we consider these charges free, then Eq. (8.4) takes the form:

$$
\operatorname{rot} \vec{H}=\vec{j}_{\Sigma}=\varepsilon_{0}\left(1-\frac{\omega^{2} p d}{\left(\omega^{2}-\omega_{0}^{2}\right)}\right) \frac{\partial \vec{E}}{\partial t} .
$$

To appears temptation to name the value 


$$
\varepsilon^{*}(\omega)=\varepsilon_{0}\left(1-\frac{\omega^{2} p d}{\left(\omega^{2}-\omega_{0}^{2}\right)}\right)
$$

by the depending on the frequency dielectric constant of dielectric. But this, as in the case conductors, cannot be made, since this is the composite parameter, which includes now those not already three depending on the frequency of the parameter: the dielectric constant of vacuum, the natural frequency of atoms or molecules and plasma frequency for the charge carriers, entering their composition.

Let us examine two limiting cases:

1. If $\omega \ll \omega_{0}$, then from (8.5) we obtain

$$
\operatorname{rot} \vec{H}=\vec{j}_{\Sigma}=\varepsilon_{0}\left(1+\frac{\omega_{p d}^{2}}{\omega_{0}^{2}}\right) \frac{\partial \vec{E}}{\partial t} .
$$

In this case the coefficient, confronting the derivative, does not depend on frequency, and it presents the static dielectric constant of dielectric. As we see, it depends on the natural frequency of oscillation of atoms or molecules and on plasma frequency. This result is intelligible. Frequency in this case proves to be such low that the charges manage to follow the field and their inertia properties do not influence electrodynamic processes. In this case the bracketed expression in the right side Eq. (8.7) presents the static dielectric constant of dielectric. As we see, it depends on the natural frequency of oscillation of atoms or molecules and on plasma frequency. Hence immediately we have a prescription for creating the dielectrics with the high dielectric constant. In order to reach this, should be in the assigned volume of space packed a maximum quantity of molecules with maximally soft connections between the charges inside molecule itself.

2. The case, when $\omega \gg \omega_{0}$

$$
\operatorname{rot} \vec{H}=\vec{j}_{\Sigma}=\varepsilon_{0}\left(1-\frac{\omega_{p d}^{2}}{\omega_{0}^{2}}\right) \frac{\partial \vec{E}}{\partial t}
$$

and dielectric became conductor (plasma), since the obtained equation exactly coincides with the equation, which describes plasma.

One cannot fail to note the circumstance that in this case again nowhere was used this concept as polarization vector, but examination is carried out by the way of finding the real currents in the dielectrics on the basis of the equation of motion of charges in these media. In this case as the parameters are used the electrical characteristics of the media, which do not depend on frequency.

From Eq. (8.5) is evident that in the case of fulfilling the equality $\omega=\omega_{0}$, the amplitude of fluctuations is equal to infinity. This indicates the presence of resonance at this point. The infinite amplitude of fluctuations occurs because of the fact that they were not considered losses in the resonance system, in this case its quality was equal to infinity. In a certain approximation it is possible to consider that lower than the point indicated we deal concerning the dielectric, whose dielectric constant is equal to its static value. Higher than this point we deal already actually concerning the metal, whose density of current carriers is equal to the density of atoms or molecules in the dielectric.

Now it is possible to examine the question of why dielectric prism decomposes polychromatic light into monochromatic components or why rainbow is formed. So that this phenomenon would occur, it is necessary to have the frequency dispersion of the phase speed of electromagnetic waves in the medium in question. If we to Eq. (8.5) add the Maxwell first equation , then we will obtain:

$$
\begin{aligned}
& \operatorname{rot} \vec{E}=-\mu_{0} \frac{\partial \vec{H}}{\partial t} \\
& \operatorname{rot} \vec{H}=\varepsilon_{0}\left(1-\frac{\omega_{p d}^{2}}{\left(\omega^{2}-\omega_{0}^{2}\right)}\right) \frac{\partial \vec{E}}{\partial t},
\end{aligned}
$$

from where we immediately find the wave equation:

$$
\nabla^{2} \vec{E}=\mu_{0} \varepsilon_{0}\left(1-\frac{\omega_{p d}{ }^{2}}{\left(\omega^{2}-\omega_{0}^{2}\right)}\right) \frac{\partial^{2} \vec{E}}{\partial t^{2}} .
$$

If one considers that

$$
\mu_{0} \varepsilon_{0}=\frac{1}{c^{2}},
$$

where $c$ is speed of light, then no longer will remain doubts about the fact that with the propagation of electromagnetic waves in the dielectrics the frequency dispersion of phase speed will be observed. But this dispersion will be connected not with the fact that this material parameter as dielectric constant, it depends on frequency. In the formation of this dispersion it will participate immediately three, which do not depend on the frequency, physical quantities: the self-resonant frequency of atoms themselves or molecules, the plasma frequency of charges, if we consider it their free, and the dielectric constant of vacuum.

Now let us show, where it is possible to be mistaken, if with the solution of the examined problem of using a concept of polarization vector. Let us introduce this polarization vector

$$
\vec{P}=-\frac{n e^{2}}{m} \cdot \frac{1}{\left(\omega^{2}-\omega_{0}^{2}\right)} \vec{E} .
$$

Its dependence on the frequency, is connected with the presence of mass in the charges, entering the constitution of atom and molecules of dielectrics. The inertness of charges is not allowed for this vector, following the electric field, to reach that value, which it would have in the permanent fields. Since the electrical induction is determined by the equation:

$$
\vec{D}=\varepsilon_{0} \vec{E}+\vec{P} \vec{E}=\varepsilon_{0} \vec{E}-\frac{n e^{2}}{m} \cdot \frac{1}{\left(\omega^{2}-\omega_{0}^{2}\right)} \vec{E},
$$

that, introduced thus, it depends on frequency.

If the vector $\vec{D}$ was introduced into the Maxwell second equation, then it will take the form: 


$$
\operatorname{rot} \vec{H}=j_{\Sigma}=\varepsilon_{0} \frac{\partial \vec{E}}{\partial t}+\frac{\partial \vec{P}}{\partial t}
$$

or

$$
\operatorname{rot} \vec{H}=j_{\Sigma}=\varepsilon_{0} \frac{\partial \vec{E}}{\partial t}-\frac{n e^{2}}{m} \frac{1}{\left(\omega^{2}-\omega_{0}^{2}\right)} \frac{\partial \vec{E}}{\partial t},
$$

where $j_{\Sigma}$ is the summed current, which flows through the model. In expression (8.9) the first member of right side presents bias current in the vacuum. The second term presents the current, which is the consequence of the presence of bound charges in atoms or molecules of dielectric. In this expression again appeared the specific kinetic inductance of the charges, which participate in the oscillating process

$$
L_{k d}=\frac{m}{n e^{2}} .
$$

This kinetic inductance determines the inductance of bound charges. Taking into account this Eq. (8.9) it is possible to rewrite

$$
\operatorname{rot} \vec{H}=j_{\Sigma}=\varepsilon_{0} \frac{\partial \vec{E}}{\partial t}-\frac{1}{L_{k d}} \frac{1}{\left(\omega^{2}-\omega_{0}^{2}\right)} \frac{\partial \vec{E}}{\partial t},
$$

Obtained expression exactly coincides with Eq. (8.3). Consequently, the eventual result of examination by both methods coincides, and there are no claims to the method from a mathematical point of view. But from a physical point of view are large claims, which we already discussed. Is certain, this not electrical induction, but the certain composite parameter. In the essence, physically substantiated is the introduction to electrical induction in the dielectrics only in the static electric fields.

Let us show that the equivalent the schematic of dielectric presents the sequential resonant circuit, whose inductance is the kinetic inductance $L_{k d}$, and capacity is equal to the static dielectric constant of dielectric minus the capacity of the equal dielectric constant of vacuum. In this case outline itself proves to be that shunted by the capacity, equal to the specific dielectric constant of vacuum. For the proof of this let us examine the sequential oscillatory circuit, when the inductance $L$ and the capacity $C$ are connected in series.

The connection between the current $I_{C}$, which flows through the capacity $C$, and the voltage $U_{C}$, applied to it, is determined by the equations:

$$
U_{C}=\frac{1}{C} \int I_{C} d t
$$

and

$$
I_{C}=C \frac{d U_{C}}{d t} .
$$

This connection will be written down for the inductance:

$$
I_{C}=\frac{1}{L} \int U_{L} d t
$$

and

$$
U_{L}=L \frac{d I_{L}}{d t}
$$

If the current, which flows through the series circuit, changes according to the law $I=I_{0} \sin \omega t$, then a voltage drop across inductance and capacity they are determined by the equations

$$
U_{L}=\omega L I_{0} \cos \omega t
$$

and

$$
U_{C}=-\frac{1}{\omega C} I_{0} \cos \omega t
$$

and total voltage applied to the outline is equal

$$
U_{\Sigma}=\left(\omega L-\frac{1}{\omega C}\right) I_{0} \cos \omega t .
$$

In this equation the value, which stands in the brackets, presents the reactance of sequential resonant circuit, which depends on frequency. The voltage, generated on the capacity and the inductance, are located in the reversed phase, and, depending on frequency, outline can have the inductive, the whether capacitive reactance. At the point of resonance the summary reactance of outline is equal to zero.

It is obvious that the connection between the total voltage applied to the outline and the current, which flows through the outline, will be determined by the equation

$$
I=-\frac{1}{\omega\left(\omega L-\frac{1}{\omega C}\right)} \frac{\partial U_{\Sigma}}{\partial t} .
$$

The resonance frequency of outline is determined by the equation

$$
\omega_{0}=\frac{1}{\sqrt{L C}}
$$

therefore

$$
I=\frac{C}{\left(1-\frac{\omega^{2}}{\omega_{0}^{2}}\right)} \frac{\partial U_{\Sigma}}{\partial t} .
$$

Comparing this expression with Eq. (8.10) it is not difficult to see that the sequential resonant circuit, which consists of the inductance $L$ and capacity $C$, it is possible to present to the capacity ofin the form dependent on the frequency

$$
C(\omega)=\frac{C}{\left(1-\frac{\omega^{2}}{\omega_{0}^{2}}\right)}
$$

This idea does not completely mean that the inductance is somewhere lost. It enters into the resonance frequency of the outline $\omega_{0}$. Equation (8.12) this altogether only the mathematical form of the record of Eq. (8.11). Consequently, this is $C(\omega)$ the certain composite mathematical parameter, which is not the capacity of outline.

Equation (8.11) can be rewritten and differently: 


$$
I=-\frac{1}{L\left(\omega^{2}-\omega_{0}^{2}\right)} \frac{\partial U_{\Sigma}}{\partial t}
$$

and to consider that

$$
C(\omega)=-\frac{1}{L\left(\omega^{2}-\omega_{0}^{2}\right)}
$$

Is certain, the parameter of $C(\omega)$, introduced in accordance with Eqs. (8.13) and (8.14) no to capacity refers.

Let us examine two limiting cases:

1. When $\omega \ll \omega_{0}$, we have

$$
I=C \frac{\partial U_{\Sigma}}{\partial t}
$$

This result is intelligible, since. at the low frequencies the reactance of the inductance, connected in series with the capacity, is considerably lower than the capacitive and it is possible not to consider it.

The equivalent the schematic of the dielectric, located between the planes of long line is shown in Figure 5.

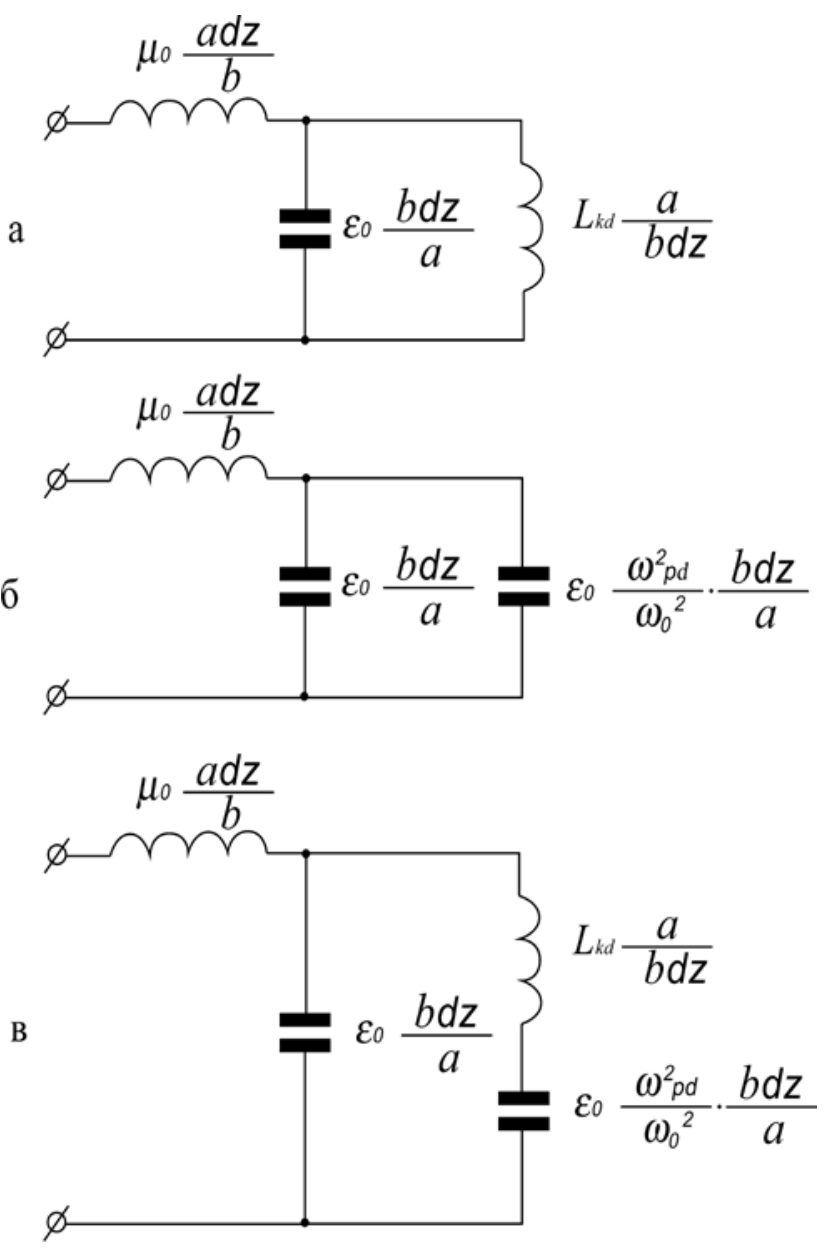

Figure 5. a - equivalent the schematic of the section of the line, filled with dielectric, for the case $\omega \gg \omega ; \sigma$ - the equivalent the schematic of the section of line for the case $\omega \ll \omega_{0}$; в - the equivalent the schematic of the section of line for entire frequency band.

2. For the case, when $\omega \gg \omega_{0}$, we have

$$
I=-\frac{1}{\omega^{2} L} \frac{\partial U_{\Sigma}}{\partial t} .
$$

Taking into account that for the harmonic signal

$$
\frac{\partial U_{\Sigma}}{\partial t}=-\omega^{2} \int U_{\Sigma} d t
$$

we will obtain from (8.15)

$$
I_{L}=\frac{1}{L} \int U_{\Sigma} d t
$$

In this case the reactance of capacity is considerably less than in inductance and chain has inductive reactance.

The carried out analysis show, that is in practice very difficult to distinguish the behavior of resonant circuits of the inductance or of the capacity. In order to understand the true composition of the chain being investigated it is necessary to remove the amplitude and phase response of this chain in the range of frequencies. In the case of resonant circuit this dependence will have the typical resonance nature, when on both sides resonance the nature of reactance is different.

In Figure 5 (a) and Figure 5 (б) are shown two limiting cases. In the first case, when $\omega \gg \omega_{0}$, dielectric according to its properties corresponds to conductor, in the second case, when $\omega \ll \omega_{0}$, it corresponds to the dielectric, which possesses the static dielectric constant

$$
\varepsilon=\varepsilon_{0}\left(1+\frac{\omega_{p d}^{2}}{\omega_{0}^{2}}\right)
$$

Thus, it is possible to make the conclusion that the introduction, the depending on the frequency dielectric constants of dielectrics, are physical and terminological error. If the discussion deals with the dielectric constant of dielectrics, with which the accumulation of potential energy is connected, then the discussion can deal only with the static permeability. And precisely this parameter as the constant, which does not depend on the frequency, enters into all equations, which characterize the electrodynamic characteristics of dielectrics.

The most interesting results of applying such new approaches occur precisely for the dielectrics. In this case each connected pair of charges presents the separate unitary unit with its individual characteristics and its participation in the processes of interaction with the electromagnetic field (if we do not consider the connection between the separate pairs) strictly individually. Certainly, in the dielectrics not all dipoles have different characteristics, but there are different groups with similar characteristics, and each group of bound charges with the identical characteristics will resound at its frequency. Moreover the intensity of absorption, and in the excited state and emission, at this frequency will depend on a relative quantity of pairs of this type. Therefore the partial coefficients, which consider their statistical weight in this process, can be introduced. Furthermore, these processes will influence the anisotropy of the dielectric properties of molecules themselves, which have the specific electrical orientation in crystal lattice. By these circumstances is determined the variety of resonances and their intensities, which is observed in the dielectric media. The lines of absorption or emission, when there is a electric coupling between the separate groups of emitters, acquire even more complex structure. In this case the lines can be converted into the strips. 


\section{PART II. New Ideas in the Classical Electrodynamics \\ or}

\section{Dynamic Potentials and the Field of the Moving Charges}

The way, which is concerned the introduction of total derivatives field on and vector potential it was begun still in Maxwell, since it wrote its equations in the total derivatives. Hertz also wrote the equations of electrodynamics in the total derivatives. Hertz did not introduce the concept of vector potentials, but he operated only with fields, but this does not diminish its merits. It made mistakes only in the fact that the electrical and magnetic fields were considered the invariants of speed. But already simple example of long lines is evidence of the inaccuracy of this approach. With the propagation of wave in the long line it is filled up with two forms of energy, which can be determined through the currents and the voltages or through the electrical and magnetic fields in the line. And only after wave will fill with electromagnetic energy all space between the generator and the load on it it will begin to be separated energy. I.e. the time, by which stays this process, generator expended its power to the filling with energy of the section of line between the generator and the load. But if we begin to move away load from incoming line, then a quantity of energy being isolated on it will decrease, since. the part of the energy, expended by source, will leave to the filling with energy of the additional length of line, connected with the motion of load. If load will approach a source, then it will obtain an additional quantity of energy due to the decrease of its length. But if effective resistance is the load of line, then an increase or the decrease of the power expendable in it can be connected only with a change in the stress on this resistance.

Being located in assigned IRF, us interest those fields, which are created in it by the fixed and moving charges, and also by the electromagnetic waves, which are generated by the fixed and moving sources of such waves. The fields, which are created in this IRF by moving charges and moving sources of electromagnetic waves, we will call dynamic. Can serve as an example of dynamic field the magnetic field, which appears around the moving charges.

As already mentioned, in the classical electrodynamics be absent the rule of the conversion of electrical and magnetic field on upon transfer of one inertial system to another. This deficiency removes SR, basis of which are the Lorenz conversions. With the entire mathematical validity of this approach the physical essence of such conversions up to now remains unexplained in this division will made attempt find the precisely physically substantiated ways of obtaining the conversions field on upon transfer of one IRF to another, and to also explain what dynamic potentials and fields can generate the moving charges. The first step in this direction was made a way of the introduction of the symmetrical laws of magnetoelectric and electromagnetic induction [5,13,14,15]. These laws are written as follows:

$$
\begin{aligned}
& \oint \vec{E}^{\prime} d l^{\prime}=-\int \frac{\partial \vec{B}}{\partial t} d \vec{s}+\oint[\vec{v} \times \vec{B}] d l^{\prime} \\
& \oint \vec{H}^{\prime} d l^{\prime}=\int \frac{\partial \vec{D}}{\partial t} d \vec{s}-\oint[\vec{v} \times \vec{D}] d l^{\prime}
\end{aligned}
$$

$$
\begin{aligned}
& \operatorname{rot} \vec{E}^{\prime}=-\frac{\partial \vec{B}}{\partial t}+\operatorname{rot}[\vec{v} \times \vec{B}] \\
& \operatorname{rot} \vec{H}^{\prime}=\frac{\partial \vec{D}}{d t}-\operatorname{rot}[\vec{v} \times \vec{D}] .
\end{aligned}
$$

For the constants fields on these equations they take the form:

$$
\begin{aligned}
& \vec{E}^{\prime}=[\vec{v} \times \vec{B}] \\
& \vec{H}^{\prime}=-[\vec{v} \times \vec{D}] .
\end{aligned}
$$

In Eqs. (9.1-9.3), which assume the validity of the Galileo conversions present fields and elements in moving and fixed IRF respectively. It must be noted, that conversions (9.3) earlier could be obtained only from the Lorenz conversions.

Equations (9.1-9.3), which present the laws of induction, do not give information about how arose fields in initial fixed IRF. They describe only laws governing the propagation and conversion fields on in the case of motion with respect to the already existing fields.

Equation (16.3) attest to the fact that in the case of relative motion of frame of references, between the fields $\vec{E}$ and $\vec{H}$ there is a cross coupling, i.e., motion in the fields $\vec{H}$ leads to the appearance field on $\vec{E}$ and vice versa. From these equations escape the additional consequences, which were for the first time examined in the article [5]. The electric field $E=\frac{g}{2 \pi \varepsilon r}$ beyond the limits of the charged long rod, where $g$ is a linear charge, diminishes according to the law $\frac{1}{r}$.

If we in parallel to the axis of rod in the field $E$ begin to move with the speed $\Delta v$ another IRF, then in it will appear the additional magnetic field $\Delta H=\varepsilon E \Delta v$. If we now with respect to already moving IRF begin to move third frame of reference with the speed $\Delta v$, then already due to the motion in the field $\Delta H$ will appear additive to the electric field $\Delta E=\mu \varepsilon E(\Delta v)^{2}$. This process can be continued and further, as a result of which can be obtained the number, which gives the value of the electric field $E_{v}^{\prime}(r)$ in moving IRF with reaching of the speed $v=n \Delta v$, when $\Delta v \rightarrow 0$, and $n \rightarrow \infty$. In the final analysis in moving IRF the value of dynamic electric field will prove to be more than in the initial and to be determined by the equation:

$$
E^{\prime}\left(r, v_{\perp}\right)=\frac{g c h \frac{v_{\perp}}{c}}{2 \pi \varepsilon r}=\operatorname{Ech} \frac{v_{\perp}}{c} .
$$

If speech goes about the electric field of the single charge $e$, then its electric field will be determined by the equation:

$$
E^{\prime}\left(r, v_{\perp}\right)=\frac{e c h \frac{v_{\perp}}{c}}{4 \pi \varepsilon r^{2}},
$$

where $v_{\perp}$ is normal component of charge rate to the vector, which connects the moving charge and observation point. 
Expression for the scalar potential, created by the moving charge, for this case will be written down as follows:

$$
\varphi^{\prime}\left(r, v_{\perp}\right)=\frac{e c h \frac{v_{\perp}}{c}}{4 \pi \varepsilon r}=\varphi(r) \operatorname{ch} \frac{v_{\perp}}{c}
$$

where $\varphi(r)$ is scalar potential of fixed charge. The potential $\varphi^{\prime}\left(r, v_{\perp}\right)$ can be named scalar-vector, since it depends not only on the absolute value of charge, but also on speed and direction of its motion with respect to the observation point. Maximum value this potential has in the direction normal to the motion of charge itself. Moreover, if charge rate changes, which is connected with its acceleration, then can be calculated the electric fields, induced by the accelerated charge.

During the motion in the magnetic field, using the already examined method, we obtain:

$$
H^{\prime}\left(v_{\perp}\right)=H \operatorname{ch} \frac{v_{\perp}}{c} .
$$

where of $v_{\perp}$ - speed normal to the direction of the magnetic field.

If we apply the obtained results to the electromagnetic wave and to designate components fields on parallel speeds IRF as $E_{\uparrow}, H_{\uparrow}$, and $E_{\perp}, H_{\perp}$ as components normal to it, then conversions fields on they will be written down:

$$
\begin{aligned}
& \vec{E}_{\uparrow}^{\prime}=\vec{E}_{\uparrow}, \\
& \vec{E}_{\perp}^{\prime}=\vec{E}_{\perp} \operatorname{ch} \frac{v}{c}+\frac{Z_{0}}{v}\left[\vec{v} \times \vec{H}_{\perp}\right] \operatorname{sh} \frac{v}{c}, \\
& \vec{H}_{\uparrow}^{\prime}=\vec{H}_{\uparrow}, \\
& \vec{H}_{\perp}^{\prime}=\vec{H}_{\perp} \operatorname{ch} \frac{v}{c}-\frac{1}{v Z_{0}}\left[\vec{v} \times \vec{E}_{\perp}\right] \operatorname{sh} \frac{v}{c},
\end{aligned}
$$

where $Z_{0}=\sqrt{\frac{\mu_{0}}{\varepsilon_{0}}}$ is impedance of free space, $c=\sqrt{\frac{1}{\mu_{0} \varepsilon_{0}}}$ is speed of light.

Conversions fields on (9.5) they were for the first time obtained in the article [5].

\section{Phase Aberration and the Transverse Doppler Effect}

Using Eqs. (9.5) it is possible to explain the phenomenon of phase aberration, which did not have within the framework existing classical electrodynamics of explanations. We will consider that there are components of the plane wave $H_{z}, E_{x}$, which is extended in the direction $y$, and primed system moves in the direction of the axis $x$ with the speed $v_{x}$. Then components fields will be written down:

$$
\begin{aligned}
& E_{x}^{\prime}=E_{x}, \\
& E_{y}^{\prime}=H_{z} \operatorname{sh} \frac{v_{x}}{c}, \\
& H_{z}^{\prime}=H_{z} \operatorname{ch} \frac{v_{x}}{c} .
\end{aligned}
$$

Thus, is a heterogeneous wave, which has in the direction of propagation the component $E_{v}^{\prime}$.

Let us write down the summary field $E^{\prime}$ in moving IRF

$$
E^{\prime}=\left[\left(E_{x}^{\prime}\right)^{2}+\left(E_{y}^{\prime}\right)^{2}\right]^{\frac{1}{2}}=E_{x} \operatorname{ch} \frac{v_{x}}{c} .
$$

If the vector $\vec{H}^{\prime}$ is as before orthogonal the axis $y$, then the vector $\vec{E}^{\prime}$ is now inclined toward it to the angle $\alpha$, determined by the equation:

$$
\alpha \cong \operatorname{sh} \frac{v}{C} \cong \frac{v}{C}
$$

This is phase aberration. Specifically, to this angle to be necessary to incline telescope in the direction of the motion of the Earth around the sun in order to observe stars, which are located in the zenith.

The Poynting vector is now also directed no longer along the axis $y$, but being located in the plane $x y$, it is inclined toward the axis $y$ to the angle, determined by Eqs. (10.2). However, the relation of the absolute values of the vectors of $\vec{E}^{\prime}$ and $\vec{H}^{\prime}$ in both systems they remained identical. However, the absolute value of Poynting vector increased. Thus, even transverse motion of inertial system with respect to the direction of propagation of wave increases its energy in the moving system. This phenomenon is understandable from a physical point of view. It is possible to give an example with the rain drops. When they fall vertically, then is energy in them one. But in the inertial system, which is moved normal to the vector of their of speed, to this speed the velocity vector of inertial system is added. In this case the absolute value of the speed of drops in the inertial system will be equal to square root of the sum of the squares of the speeds indicated. The same result gives to us Eq. (10.1).

Such waves have in the direction of its propagation additional of the vector of electrical or magnetic field, and in this they are similar to $E$ and $H$ of the waves, which are extended in the waveguides. In this case appears the uncommon wave, whose phase front is inclined toward the Poynting vector to the angle, determined by Eq. (10.2). In fact obtained wave is the superposition of plane wave with the phase speed $c=\sqrt{\frac{1}{\mu \varepsilon}}$ and additional wave of plane wave with the infinite phase speed orthogonal to the direction of propagation.

The transverse Doppler effect, who long ago is discussed sufficiently, until now, did not find its confident experimental confirmation. For observing the star from moving IRF it is necessary to incline telescope on the motion of motion to the angle, determined by Eq. (10.2). But in this case the star, observed with the aid of the telescope in the zenith, will be in actuality located several behind the visible position with respect to the direction of motion. Its angular displacement from the visible position in this case will be determined by Eq. (10.2). But this means that this star with respect to the observer has radial spid, determined by the equation

$$
v_{r}=v \sin \alpha .
$$


Since for the low values of the angles $\sin \alpha \cong \alpha$, and $\alpha=\frac{v}{c}$, Doppler frequency shift will compose

$$
\omega_{d \perp}=\omega_{0} \frac{v^{2}}{c^{2}} .
$$

This result numerically coincides with results SR, but it is principally characterized by of results. It is considered SR that the transverse Doppler effect, determined by Eq. (10.3), there is in reality, while in this case this only apparent effect. If we compare the results of conversions fields on (10.5) with conversions SP, then it is not difficult to see that they coincide with an accuracy to the quadratic members of the ratio of the velocity of the motion of charge to the speed of light.

Conversion SP, although they were based on the postulates, could correctly explain sufficiently accurately many physical phenomena, which before this explanation did not have. With this circumstance is connected this great success of this theory. Conversions (10.4) and (10.5) are obtained on the physical basis without the use of postulates and they with the high accuracy coincided with SP. Difference is the fact that in conversions (10.5) there are no limitations on the speed for the material particles, and also the fact that the charge is not the invariant of speed. The experimental confirmation of the fact indicated can serve as the confirmation of correctness of the proposed conversions.

\section{The Problem of the Lorentz Force and Power Interaction of the current-Carrying Systems and Its Solution}

It was already said, that Maxwell equations do not include information about power interaction of the current carrying systems. In the classical electrodynamics for calculating such an interaction it is necessary to calculate magnetic field in the assigned region of space, and then, using a Lorentz force, to find the forces, which act on the moving charges. Obscure a question about that remains with this approach, to what are applied the reacting forces with respect to those forces, which act on the moving charges.

The concept of magnetic field arose to a considerable degree because of the observations of power interaction of the current carrying and magnetized systems. Experience with the iron shavings, which are erected near the magnet poles or around the annular turn with the current into the clear geometric figures, is especially significant. These figures served as occasion for the introduction of this concept as the lines of force of magnetic field. In accordance with third Newton law with any power interaction there is always a equality of effective forces and opposition, and also always there are those elements of the system, to which these forces are applied. A large drawback in the concept of magnetic field is the fact that it does not give answer to that, counteracting forces are concretely applied to what, since. magnetic field comes out as the independent substance, with which occurs interaction of the moving charges.

Is experimentally known that the forces of interaction in the current carrying systems are applied to those conductors, whose moving charges create magnetic field. However, in the existing concept of power interaction of such systems the positively charged lattice, to which are applied the forces, does not participate in the formation of the forces of interaction.

Let us examine this question on the basis of the concept of scalar- vector potential. We will consider that the scalar- vector potential of single charge is determined by Eq. (9.4), and that the electric fields, created by this potential, act on all surrounding charges, including to the charges positively charged lattices.

Let us examine from these positions power interaction between two parallel conductors (Figure 6), along which flow the currents. We will consider that $g_{1}{ }^{+}, g_{2}{ }^{+}$and $g_{1}{ }^{-}, g_{2}{ }^{-}$present the respectively fixed and moving linear charges.

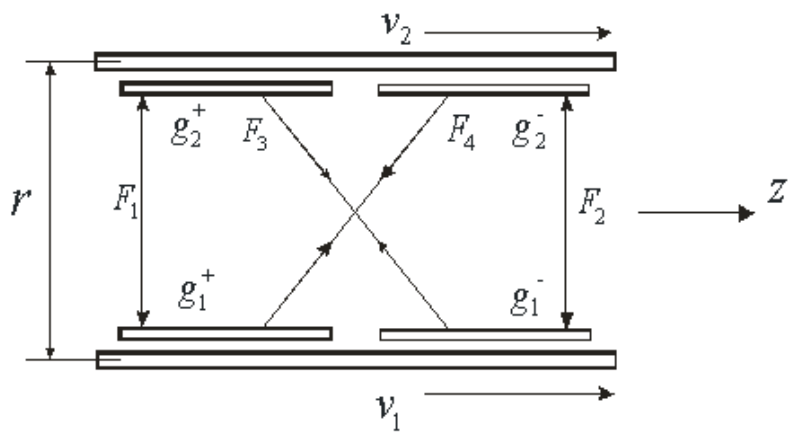

Figure 6. Schematic of power interaction of the current carrying wires of two-wire circuit taking into account the positively charged lattice

The linear charges $g_{1}^{+}, g_{2}{ }^{+}$present the positively charged lattice in the lower and upper conductors. We will also consider that both conductors prior to the start of charges are electrically neutral. This means that in the conductors are two systems of the mutually inserted opposite charges with the linear density $g_{1}^{+}, g_{1}^{-}$and $g_{2}{ }^{+}, g_{2}{ }^{-}$, which neutralize each other. In Figure 6 these systems for larger convenience in the examination of the forces of interaction are moved apart along the axis $z$. Subsystems with the negative charge (electrons) can move with the speeds $v_{1}, v_{2}$. The force of interaction between the lower and upper conductors we will search for as the sum of four forces, whose designation is understandable from the figure. The repulsive forces $F_{1}, F_{2}$ we will take with the minus sign, while the attracting force $F_{3}, F_{4}$ we will take with the plus sign.

For the single section of the two-wire circuit of force, acting between the separate subsystems, will be written down

$$
\begin{aligned}
& F_{1}=-\frac{g_{1}{ }^{+} g_{2}{ }^{+}}{2 \pi \varepsilon r}, \\
& F_{2}=-\frac{g_{1}{ }^{-} g_{2}{ }^{-}}{2 \pi \varepsilon r} \operatorname{ch} \frac{v_{1}-v_{2}}{c}, \\
& F_{3}=+\frac{g_{1}^{-} g_{2}{ }^{+}}{2 \pi \varepsilon r} \operatorname{ch} \frac{v_{1}}{c}, \\
& F_{4}=+\frac{g_{1}{ }^{+} g_{2}{ }^{-}}{2 \pi \varepsilon r} \operatorname{ch} \frac{v_{2}}{c} .
\end{aligned}
$$


Adding all force components, we will obtain the amount of the composite linear

force

$$
F_{\Sigma}=\frac{g_{1} g_{2}}{2 \pi \varepsilon r}\left(\operatorname{ch} \frac{v_{1}}{c}+\operatorname{ch} \frac{v_{2}}{c}-\operatorname{ch} \frac{v_{1}-v_{2}}{c}-1\right) .
$$

In this expression as $g_{1}$ and $g_{2}$ are undertaken the absolute values of the linear charges, and the signs of forces are taken into account in the bracketed expression. For the case $v \ll c$ let us take only two first members of expansion in the series $\operatorname{ch} \frac{v}{c}$, i.e., we will consider that $\operatorname{ch} \frac{v}{c} \cong 1+\frac{1}{2} \frac{v^{2}}{c^{2}}$. From Eq. (11.2) we obtain

$$
F_{\Sigma 1}=\frac{g_{1} v_{1} g_{2} v_{2}}{2 \pi \varepsilon c^{2} r}=\frac{I_{1} I_{2}}{2 \pi \varepsilon c^{2} r}
$$

where as $g_{1}$ and $g_{2}$ are undertaken the absolute values of the linear charges, and $v_{1}$ and $v_{2}$ take with its signs.

Since the magnetic field of straight wire, along which flows the current $I$, we determine by the equation

$$
H=\frac{I}{2 \pi r},
$$

from Eq. (11.3) we obtain

$$
F_{\Sigma 1}=\frac{I_{1} I_{2}}{2 \pi \varepsilon c^{2} r}=\frac{H_{1} I_{2}}{\varepsilon c^{2}}=I_{2} \mu H_{1},
$$

where $H_{1}$ is the magnetic field, created by lower conductor in the location of upper conductor.

It is analogous

$$
F_{\Sigma 1}=I_{1} \mu H_{2}
$$

where $\mathrm{H}_{2}$ is the magnetic field, created by upper conductor in the region of the arrangement of lower conductor.

These equations coincide with the results, obtained on the basis of the concept of magnetic field and Lorentz forces.

Equation (11.3) represents the known rule of power interaction of the current-carrying systems, but it is obtained not on the basis the introduction of phenomenological magnetic field, but on the basis of completely intelligible physical procedures. In the formation of the forces of interaction in this case the lattice takes direct part, which is not in the model of magnetic field. In the model examined are well visible the places of application of force. The obtained equations coincide with the results, obtained on the basis of the concept of magnetic field and by the axiomatically introduced Lorentz force. In this case is undertaken only first member of expansion in the series $\operatorname{ch} \frac{v}{c}$. For the speeds $v \sim c$ should be taken all terms of expansion. If we consider this circumstance, then the connection between the forces of interaction and the charge rates proves to be nonlinear. This, in particular it leads to the fact that the law of power interaction of the current-carrying systems is asymmetric. With the identical values of currents, but with their different directions, the attracting forces and repulsion become unequal. Repulsive forces prove to be greater than attracting force. This difference is small and is determined by the expression

$$
\Delta F=\frac{v^{2}}{2 c^{2}} \frac{I_{1} I_{2}}{2 \pi \varepsilon c^{2} \varepsilon},
$$

but with the speeds of the charge carriers of close ones to the speed of light it can prove to be completely perceptible.

Let us remove the lattice of upper conductor, after leaving only free electronic flux. In this case will disappear the forces of $F_{1}, F_{3}$, and this will indicate interaction of lower conductor with the flow of the free electrons, which move with the speed $v_{2}$ on the spot of the arrangement of upper conductor. In this case the value of the force of interaction is defined as:

$$
F_{\Sigma}=\frac{g_{1} g_{2}}{2 \pi \varepsilon r}\left(\operatorname{ch} \frac{v_{2}}{c}-\operatorname{ch} \frac{v_{1}-v_{2}}{c}\right) .
$$

Lorentz force assumes linear dependence between the force, which acts on the charge, which moves in the magnetic field, and his speed. However, in the obtained equation the dependence of the amount of force from the speed of electronic flux will be nonlinear. From Eq. (1.4) see that with an increase in $v_{2}$ the deviation from the linear law increases, and in the case, when $v_{2} \gg v_{1}$, the force of interaction are approached zero. This is meaningful result. Specifically, this phenomenon observed in their known experiments Thompson and Kauffmann, when they noted that with an increase in the velocity of electron beam it is more badly slanted by magnetic field. They connected the results of their observations with an increase in the mass of electron. As we see reason here another.

Let us note still one interesting result. From Eq. (11.3) the force of interaction of electronic flux with a straight wire to determine according to the following dependence:

$$
F_{\Sigma}=\frac{g_{1} g_{2}}{2 \pi \varepsilon r}\left(\frac{v_{1} v_{2}}{c^{2}}-\frac{1}{2} \frac{v_{1}^{2}}{c^{2}}\right)
$$

From Eq. (18.5) follows that with the unidirectional electron motion in the conductor and in the electronic flux the force of interaction with the fulfillment of conditions of $v_{1}=\frac{1}{2} v_{2}$ is absent.

Since the speed of the electronic flux usually much higher than speed of current carriers in the conductor, the second term in the brackets in Eq. (11.5) can be disregarded. Then, since

$$
H_{1}=\frac{g_{1} v_{1}}{2 \pi \varepsilon c^{2} r}
$$

we will obtain the magnetic field, created by lower conductor in the place of the motion of electronic flux:

$$
F_{\Sigma}=\frac{g_{1} g_{2}}{2 \pi \varepsilon r} \frac{v_{1} v_{2}}{c^{2}}=g_{2} \mu v_{2} H
$$

In this case, the obtained value of force coincides with the value of Lorentz force. 
Taking into account that

$$
F_{\Sigma}=g_{2} E=g_{2} \mu v_{2} H,
$$

it is possible to consider that on the charge, which moves in the magnetic field, acts the electric field $E$, directed normal to the direction of the motion of charge. This result also with an accuracy to of the quadratic terms $\frac{v^{2}}{c^{2}}$ completely coincides with the results of the concept of magnetic field and is determined Lorentz force.

As was already said, one of the important contradictions to the concept of magnetic field is the fact that two parallel beams of the like charges, which are moved with the identical speed in one direction, must be attracted. In this model there is no this contradiction already. If we consider that the charge rates in the upper and lower wire will be equal, and lattice is absent, i.e., to leave only electronic fluxes, then will remain only the repulsive force $F_{2}$.

Thus, the moving electronic flux interacts simultaneously both with the moving electrons in the lower wire and with its lattice, and the sum of these forces of interaction it is called Lorentz force.

Regularly does appear a question, and does create magnetic field most moving electron stream of in the absence compensating charges of lattice or positive ions in the plasma? The diagram examined shows that the effect of power interaction between the current carrying systems requires in the required order of the presence of the positively charged lattice. Therefore most moving electronic flux cannot create that effect, which is created during its motion in the positively charged lattice.

Let us demonstrate still one approach to the problem of power interaction of the current carrying systems. The statement of facts of the presence of forces between the current carrying systems indicates that there is some field of the scalar potential, whose gradient ensures the force indicated. But that this for the field? Equation (11.3) gives only the value of force, but he does not speak about that, the gradient of what scalar potential ensures these forces. We will support with constants the currents $I_{1}, I_{2}$, and let us begin to draw together or to move away conductors. The work, which in this case will be spent, and is that potential, whose gradient gives force. After integrating Eq. (11.3) on $r$, we obtain the value of the energy:

$$
W=\frac{I_{1} I_{2} \ln r}{2 \pi \varepsilon c^{2}} .
$$

This energy, depending on that to move away conductors from each other, or to draw together, can be positive or negative. When conductors move away, then energy is positive, and this means that, supporting current in the conductors with constant, generator returns energy. This phenomenon is the basis the work of all electric motors. If conductors converge, then work accomplish external forces, on the source, which supports in them the constancy of currents. This phenomenon is the basis the work of the mechanical generators of e.m.f.

Equation for the energy can be rewritten and thus:

$$
W=\frac{I_{1} I_{2} \ln r}{2 \pi \varepsilon c^{2}}=I_{2} A_{z 1}=I_{1} A_{z 2},
$$

where

$$
A_{\mathrm{z} 1}=\frac{I_{1} \ln r}{2 \pi \varepsilon c^{2}}
$$

is $Z$ component of vector potential, created by lower conductor in the location of upper conductor, and

$$
A_{z 2}=\frac{I_{2} \ln r}{2 \pi \varepsilon c^{2}}
$$

is $\mathrm{Z}$ component of vector potential, created by upper conductor in the location of lower conductor.

The approach examined demonstrates that large role, which the vector potential in questions of power interaction of the current-carrying systems and conversion of electrical energy into the mechanical plays. This approach also clearly indicates that the Lorentz force is a consequence of interaction of the current-carrying systems with the field of the vector potential, created by other current-carrying systems. Important circumstance is the fact that the formation of vector potential is obliged to the dependence of scalar potential on the speed. This is clear from a physical point of view. The moving charges, in connection with the presence of the dependence of their scalar potential on the speed, create the scalar field, whose gradient gives force. But the creation of any force field requires expenditures of energy. These expenditures accomplishes generator, creating currents in the conductors. In this case in the surrounding space is created the special field, which interacts with other moving charges according to the special vector rules. In this case only scalar product of the charge rate and vector potential gives the potential, whose gradient gives the force, which acts on the moving charge. This is the Lorentz force.

In spite of simplicity and the obviousness of this approach, this simple mechanism up to now was not finally realized. For this reason the Lorentz force, until now, was introduced in the classical electrodynamics by axiomatic way.

Let us examine the still one case, when the single negative charge $e$ moves with the speed $v_{2}$ in parallel to the conductor, along which with the speed $v_{1}$ move the electrons (Figure 15). We will consider that the conductor prior to the beginning of electron motion was electrically neutral and the linear density of positive ions and electrons they were equal. The element of the effective force of the moving charge $e$ on the element $q_{1}^{-} d z$ will be determined by the equation:

$$
d F=\frac{e g_{1} d z}{4 \pi \varepsilon r^{2}}\left(\frac{v_{1 n} v_{2 n}}{c^{2}}-\frac{1}{2} \frac{v_{1 n^{2}}}{c^{2}}\right),
$$

where $v_{1 n}, v_{2 n}$ are components of the corresponding speeds, normal to the radius, which connects the moving charge with the grain $q_{1}{ }^{-} d z$. The speed of the electron motion $v_{2 n}$ is considerably more than the speed of the motion of charges in the conductor $v_{2 n}$, therefore last term in the brackets in this equation can be disregarded.

Since of $v_{1 n}=v_{1} \sin \alpha$ and $v_{2 n}=v_{2} \sin \alpha$, and also, taking into account that 


$$
r_{0}=r \sin \alpha \text { and } d z=\frac{r_{0} d \alpha}{\sin ^{2} \alpha},
$$

we obtain

$$
d F=\frac{q_{1} v_{1} e v_{2}}{4 \pi \varepsilon c^{2} r_{0}} d \alpha
$$

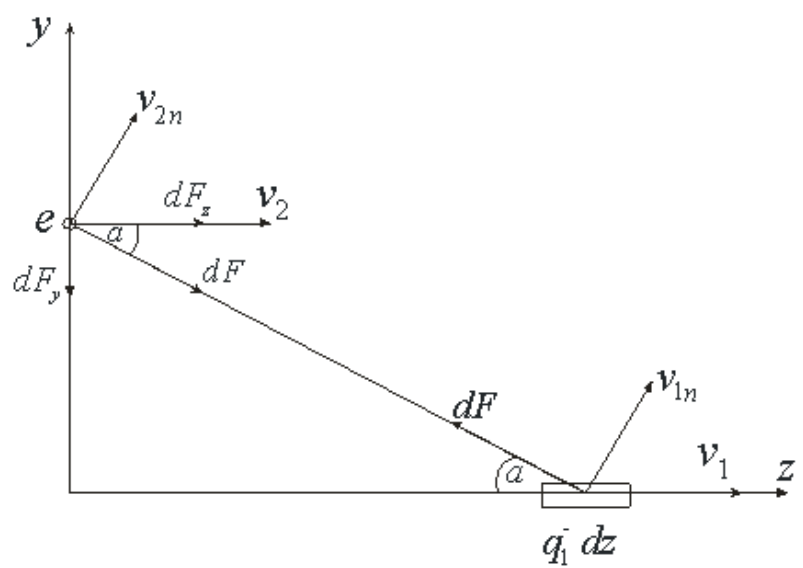

Figure 7. The diagram of interaction of the moving point charge with the conductor, along which flows the current

The obtained force corresponds to attraction. The element of this force, parallel $r_{0}$, will be written down as:

$$
d F_{y}=\frac{q_{1} v_{1} e v_{2}}{4 \pi \varepsilon c^{2} r_{0}} \sin \alpha d \alpha
$$

and the element of the force, normal to $r_{0}$ will be equal:

$$
d F_{X}=\frac{q_{1} v_{1} e v_{2}}{4 \pi \varepsilon c^{2} r_{0}} \cos \alpha d \alpha
$$

After integrating Eq. (11.6) and taking into account that the current, which flows by the lower conductor it is determined by the equation $I=q_{1} v_{1}$, let us write down the force, which acts on the single moving charge $e$ from the side of the right side of the wire:

$$
F=\int_{0}^{\frac{\pi}{2}} \frac{I e v_{2}}{4 \pi \varepsilon c^{2} r_{0}} \sin \alpha d \alpha=\frac{I e v_{2}}{4 \pi \varepsilon c^{2} r_{0}} .
$$

If we consider interaction, also, with her left side of the wire, then the force, which acts in parallel $r_{0}$ will be doubled, and the forces, which act normal to $r_{0}$, they are compensated. Thus, the composite force, which acts on the charge, which moves in parallel to wire, will be written down:

$$
F_{\Sigma}=\frac{I e v_{2}}{2 \pi \varepsilon c^{2} r_{0}}
$$

Since the magnetic field, created by lower conductor with the current at the point of the presence of the moving charge, is determined by the equation

$$
H=\frac{I}{2 \pi r_{0}},
$$

and magnetic permeability is $\mu=\frac{1}{\varepsilon c^{2}}$, then from Eq. (11.8) we obtain

$$
F_{\Sigma}=e v_{2} \mu H
$$

This force is exactly equal to Lorentz force.

Let us examine the case, when the charge moves between two limitless parallel plates, along which flows the specific current $I$, (Figure 8). This current flows along the normal to the plane of figure. In this case the charge moves in parallel to the current, which flows in the plates.

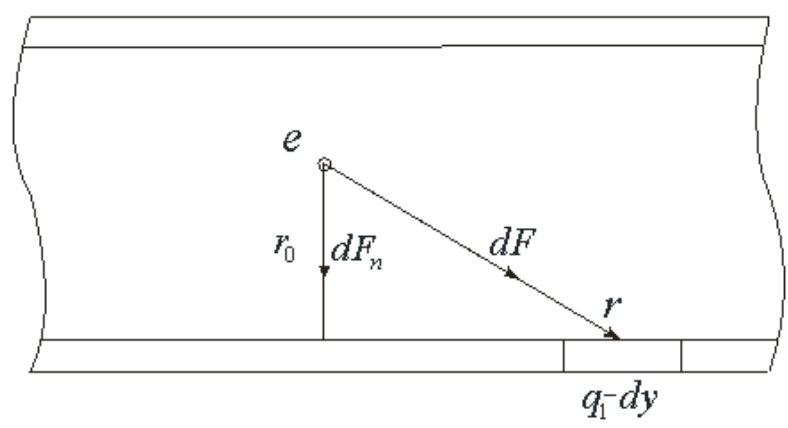

Figure 8. Diagram of interaction of the moving point charge with the currents, which flow along the parallel conducting plates

Taking into account Eq. (11.9), let us write down the element of the force, which acts on the moving charge from the side of the current element, which flows normal to the element, $d y$

$$
d F=\frac{d z d y n v_{1} q_{2} v_{2}}{2 \pi \varepsilon c^{2} r} .
$$

In this equation $d z$ is this thickness of the layer, along which the current flows, and $n$ is electron density.

Let us rewrite Eq. (11.10), taking into account that $d y=\frac{r_{0} d \alpha}{\sin ^{2} \alpha}, r=\frac{r_{0}}{\sin \alpha}$, and also that that $\frac{d F_{n}}{d F}=\sin \alpha$, where $d F$ is element of force, directed in parallel $r$, and $d F_{n}$ is element of force, directed normal to $r_{0}$ :

$$
d F_{n}=\frac{d z n v_{1} g_{2} v_{2} d \alpha}{2 \pi \varepsilon_{0} c^{2}}
$$

After integrating this eqution, we will obtain the total force, which acts on the moving charge from the side of one half-plane:

$$
F=\int_{0}^{\frac{\pi}{2}} \frac{d z n v_{1} g_{2} v_{2} d \alpha}{2 \pi \varepsilon_{0} c^{2}}=\frac{d z n v_{1} g_{2} v_{2}}{4 \varepsilon_{0} c^{2}}
$$

Taking into account that the fact that on the charge act the forces from the side of four half-planes (two from the side of lower plate two from the side of upper), finally we obtain:

$$
F_{\Sigma}=\frac{g_{2} v_{2} H}{\varepsilon_{0} c^{2}}=\mu g_{2} v_{2} H .
$$


And again eventual result exactly coincided with the results of the concept of magnetic field.

Thus, the results, obtained taking into account the introduction of scalar-vector potential and concept of magnetic field, completely coincide, if we consider only quadratic members of the expansion of hyperbolic cosine in series. In the case of the calculation of the terms of the expansion of the higher orders, when the speeds of the motion of charges are great, this agreement it will not be and the connection between the force and the speed becomes nonlinear, and the concept of magnetic field will no longer give correct results.

By the merit of this method of examining interaction between the current carrying systems and the charges appears the fact that he indicates the concrete places of application of force, which act between their elements and moving charges, which is not in the concept of magnetic field.

Now it is possible to verify does work the mechanism of interaction of the current-carrying systems in the case of the long line (Figure 2) examined. The tension of the electric field between the planes of line is determined by the equation:

$$
E=\frac{g_{\square}}{\varepsilon_{0}}
$$

$g_{\square}$ is the charge, which falls to the single square of the surface of long line.

The specific current, which falls per unit of the width of line, magnetic and electric field in it are connected with the equation

$$
I=g_{\square} v=H=\frac{E}{Z_{0}} .
$$

From this equation we obtain

$$
v=\frac{E}{g_{\square} Z_{0}} .
$$

Since the currents in the planes of line are directed in opposite directions, taking into account Eqs. (11.11 11.13), value of the repulsive force, falling to the single square surface, let us write down:

$$
F_{\square}=\frac{g_{\square}{ }^{2} v^{2}}{2 \varepsilon_{0} c^{2}}=\frac{1}{2} \mu_{0} H^{2} .
$$

Thus, the concept of scalar-vector potential and in this case gives correct answer.

Let us examine the still one interesting consequence, which escapes from the given examination. If we as the planes of long line use an superconductor, then the magnetic field on its surface, equal to specific current, can be determined from the equation:

$$
H=n e v \lambda,
$$

where $\lambda=\sqrt{\frac{m}{n e^{2} \mu}}$ is depth of penetration of magnetic field into the superconductor.

If we substitute the value of depth of penetration into Eq. (11.14), then we will obtain the unexpected result:

$$
H=v \sqrt{\frac{n m}{\mu}} .
$$

Occurs that the magnetic field strength completely does not depend on the magnitude of the charge of current carriers, but it depends on their mass. Thus, the specific energy of magnetic field on

$$
W_{H}=\frac{1}{2} \mu H^{2}=\frac{n m v^{2}}{2}
$$

is equal to specific kinetic the kinetic energy of charges. But magnetic field exists not only on its surface, also, in the skin-layer. Volume, occupied by magnetic fields, incommensurably larger than the volume of this layer. If we designate the length of the line, depicted in Fig. 2 as $l$, then the volume of skin-layer in the superconductive planes of line will compose $2 l b \lambda$. Energy of magnetic field on in this volume we determine from the equation:

$$
W_{H, \lambda}=n m v^{2} l b \lambda,
$$

however, energy of magnetic field on, accumulated between the planes of line, it will comprise:

$$
W_{H, a}=\frac{n m v^{2} l b a}{2}=\frac{1}{2} l b a \mu_{0} H .
$$

If one considers that the depth of penetration of magnetic field in the superconductors composes several hundred angstroms, then with the macroscopic dimensions of line it is possible to consider that the total energy of magnetic field on in it they determine by Eq. (11.16). Therefore, the formation of magnetic field $H$ between the planes of line, which appear in connection with the motion of charges in the skin-layer, it requires the same expenditures of energy, as if entire volume of line was filled with the particles, which move with the speed $v$, whose density and mass compose respectively $n$ and $m$.

Is obvious that the effective mass of electron in comparison with the mass of free electron grows in this case into $\frac{a}{2 \lambda}$ of times. This is the consequence of the fact that the mechanical electron motion leads not only to the accumulation of their kinetic energy in the skin-layer, but in the line also occurs accumulation and potential energies, whose gradient gives the force, which acts on the conducting planes of line. Thus, becomes clear nature of such parameters as inductance and the effective mass of electron, which in this case depend, in essence, not from the mass of free electrons, but from the configuration of conductors, on which the electrons move.

\section{Problem of Emission of Electromagnetic Wave and the Laws of the Electro- electrical Induction}

Since field on any process of the propagation of electrical and potentials it is always connected with the delay, let us introduce the being late scalar- vector potential, by considering that the field of this potential is extended in this medium with a speed of light $[13,14,15]$ : 


$$
\varphi(r, t)=\frac{g c h \frac{v_{\perp}\left(t-\frac{r}{c}\right)}{c}}{4 \pi \varepsilon_{0} r}
$$

where $v_{\perp}\left(t-\frac{r}{c}\right)$ is component of the charge rate of $g$, normal to the vector $\vec{r}$ at the moment of the time of $t^{\prime}=t-\frac{r}{c}, r$ is distance between the charge and the point, at which is determined the field, at the moment of the time $t$.

Using a equation $\vec{E}=-\operatorname{grad} \varphi(r, t)$, let us find field at point 1 (Figure 9). The gradient of the numerical value of a radius of the vector of $\vec{r}$ is a scalar function of two points: the initial point of a radius of vector and its end point (in this case this point 1 on the axis of $x$ and point 0 at the origin of coordinates). Point 1 is the point of source, while point 0 - by observation point. With the determination of gradient from the function, which contains a radius depending on the conditions of task it is necessary to distinguish two cases:

1. The point of source is fixed and is considered as the function of the position of observation point.

2. Observation point is fixed and is considered as the function of the position of the point of source.

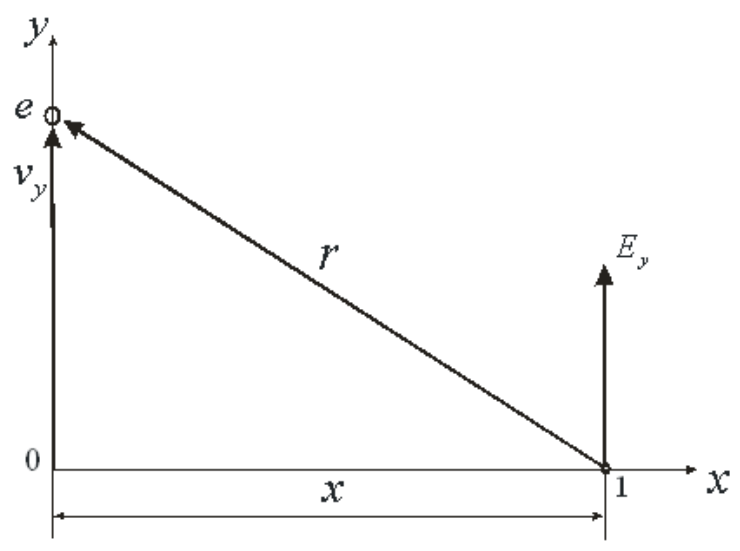

Figure 9. Diagram of shaping of the induced electric field.

We will consider that the charge $e$ accomplishes fluctuating motion along the axis $y$, in the environment of point 0 , which is observation point, and fixed point 1 is the point of source and $\vec{r}$ is considered as the function of the position of charge. Then we write down the value of electric field at point 1 :

$$
E_{y}(1)=-\frac{\partial \varphi_{\perp}(r, t)}{\partial y}=-\frac{\partial}{\partial y} \frac{e}{4 \pi \varepsilon_{0} r(y, t)} \operatorname{ch} \frac{v_{y}\left(t-\frac{r(y, t)}{c}\right)}{c}
$$

when the amplitude of the fluctuations of charge is considerably less than distance to the observation point, it is possible to consider a radius vector constant. In this case we obtain:

$$
E_{y}(x, t)=-\frac{e}{4 \pi \varepsilon_{0} c x} \frac{\partial v_{y}\left(t-\frac{x}{c}\right)}{\partial y} \operatorname{sh} \frac{v_{y}\left(t-\frac{x}{c}\right)}{c}
$$

where $x$ is some fixed point on the axis $x$.

Taking into account that

$$
\frac{\partial v_{y}\left(t-\frac{x}{c}\right)}{\partial y}=\frac{\partial v_{y}\left(t-\frac{x}{c}\right)}{\partial t} \frac{\partial t}{\partial y}=\frac{\partial v_{y}\left(t-\frac{x}{c}\right)}{\partial t} \frac{1}{v_{y}\left(t-\frac{x}{c}\right)}
$$

we obtain from (12.2):

$$
\begin{aligned}
& E_{y}(x, t) \\
& =\frac{e}{4 \pi \varepsilon_{0} c x} \frac{1}{v_{y}\left(t-\frac{x}{c}\right)} \frac{\partial v_{y}\left(t-\frac{x}{c}\right)}{\partial t} \operatorname{sh} \frac{v_{y}\left(t-\frac{x}{c}\right)}{c} .
\end{aligned}
$$

This is a complete emission law of the moving charge.

If we take only first term of the expansion, then we will obtain from (12.3):

$E_{y}(x, t)=-\frac{e}{4 \pi \varepsilon_{0} c^{2} x} \frac{\partial v_{y}\left(t-\frac{x}{c}\right)}{\partial t}=-\frac{e a_{y}\left(t-\frac{x}{c}\right)}{4 \pi \varepsilon_{0} c^{2} x}$

where $a_{y}\left(t-\frac{x}{c}\right)$ is being late acceleration of charge. This equation is wave equation and defines both the amplitude and phase responses of the wave of the electric field, radiated by the moving charge.

If we as the direction of emission take the vector, which lies at the plane $x y$, and which constitutes with the axis $y$ the angle $\alpha$, then Eq. (12.4) takes the form:

$$
E_{y}(x, t, \alpha)=-\frac{e a_{y}\left(t-\frac{x}{c}\right) \sin \alpha}{4 \pi \varepsilon_{0} c^{2} x} .
$$

Equation (12.5) determines the radiation pattern. Since in this case there is axial symmetry relative to the axis $y$, it is possible to calculate the complete radiation pattern of this emission. This diagram corresponds to the radiation pattern of dipole emission.

Since of $\frac{e v_{z}\left(t-\frac{x}{c}\right)}{4 \pi x}=A_{H}\left(t-\frac{x}{c}\right)$ is being late vector potential, Eq. (12.5) it is possible to rewrite

$$
\begin{aligned}
& E_{y}(x, t, \alpha)=-\frac{e a_{y}\left(t-\frac{x}{c}\right) \sin \alpha}{4 \pi \varepsilon_{0} c^{2} x} \\
& =-\frac{1}{\varepsilon_{0} c^{2}} \frac{\partial A_{H}\left(t-\frac{x}{c}\right)}{\partial t}=-\mu_{0} \frac{\partial A_{H}\left(t-\frac{x}{c}\right)}{\partial t}
\end{aligned}
$$

Is again obtained complete agreement with the equations of the being late vector potential, but vector potential is introduced here not by phenomenological method, but with the use of a concept of the being late scalar-vector potential. It is necessary to note one important circumstance: in Maxwell's equations the 
electric fields, which present wave, vortex. In this case the electric fields bear gradient nature.

Let us demonstrate the still one possibility, which opens Eq. (12.5). It is known that in the electrodynamics there is this concept, as the electric dipole and dipole emission. Two charges with the opposite signs have the dipole moment:

$$
\vec{p}=e \vec{d}
$$

where the vector $\vec{d}$ is directed from the negative charge toward the positive charge. Therefore current can be expressed through the derivative of dipole moment on the time

Consequently

$$
e \vec{v}=e \frac{\partial \vec{d}}{\partial t}=\frac{\partial \vec{p}}{\partial t}
$$

$$
\vec{v}=\frac{1}{e} \frac{\partial \vec{p}}{\partial t}
$$

and

$$
\vec{a}=\frac{\partial \vec{v}}{\partial t}=\frac{1}{e} \frac{\partial^{2} \vec{p}}{\partial t^{2}}
$$

Substituting this equation into expression (12.5), we obtain the emission law of the being varied dipole.

$$
\vec{E}=-\frac{1}{4 \pi r \varepsilon_{0} c^{2}} \frac{\partial^{2} p\left(t-\frac{r}{c}\right)}{\partial t^{2}}
$$

This is also known equation [1].

In the process of fluctuating the electric dipole are created the electric fields of two forms. First, these are the electrical induction fields of emission, represented by equations (12.4), (12.5) and (12.6), connected with the acceleration of charge. In addition to this, around the being varied dipole are formed the electric fields of static dipole, which change in the time in connection with the fact that the distance between the charges it depends on time. These fields present the fields of the neighbor zone of dipole source. Specifically, energy of these field on the freely being varied dipole and it is expended on the emission. However, the summary value of field around this dipole at any moment of time defines as superposition fields on static dipole field on emissions.

The laws (12.4), (12.5), (12.7) are the laws of the direct action, in which already there is neither magnetic field on nor vector potentials. I.e. those structures, by which there were the magnetic field and magnetic vector potential, are already taken and they no longer were necessary to us.

Using Eq. (12.5) it is possible to obtain the laws of reflection and scattering both for the single charges and, for any quantity of them. If any charge or group of charges undergo the action of external (strange) electric field, then such charges begin to accomplish a forced motion, and each of them emits electric fields in accordance with Eq. (12.5). The superposition of electrical field on, radiated by all charges, it is electrical wave.

If on the charge acts the electric field of , then the acceleration of charge is determined by the equation

$$
a=-\frac{e}{m} E_{y 0}^{\prime} \sin \omega t
$$

Taking into account this Eq. (12.5) assumes the form

$$
\begin{aligned}
& E_{y}(x, t, \alpha)=\frac{e^{2} \sin \alpha}{4 \pi \varepsilon_{0} c^{2} m x} E_{y 0}^{\prime} \sin \omega\left(t-\frac{x}{c}\right) \\
& =\frac{K}{x} E_{y 0}^{\prime} \sin \omega\left(t-\frac{x}{c}\right)
\end{aligned}
$$

where the coefficient $K=\frac{e^{2} \sin \alpha}{4 \pi \varepsilon_{0} c^{2} m}$ can be named the coefficient of scattering (re-emission) single charge in the assigned direction, since it determines the ability of charge to re-emit the acting on it external electric field.

The current wave of the displacement accompanies the wave of electric field:

$$
j_{y}(x, t)=\varepsilon_{0} \frac{\partial E_{y}}{\partial t}=-\frac{e \sin \alpha}{4 \pi c^{2} x} \frac{\partial^{2} v_{y}\left(t-\frac{x}{c}\right)}{\partial t^{2}} .
$$

If charge accomplishes its motion under the action of the electric field $E^{\prime}=E_{0}^{\prime} \sin \omega t$, then bias current in the distant zone will be written down as

$$
j_{y}(x, t)=-\frac{e^{2} \omega}{4 \pi c^{2} m x} E_{y 0}^{\prime} \cos \omega\left(t-\frac{x}{c}\right) .
$$

The sum wave, which presents the propagation of electrical field on (12.8) and bias currents (12.9), can be named electrocurent wave. In this current wave of displacement lags behind the wave of electric field to the angle equal $\frac{\pi}{2}$. For the first time this term and definition of this wave was used in the articles [13,15].

In parallel with the electrical waves it is possible to introduce magnetic waves, if we assume that

$$
\begin{gathered}
\vec{j}=\varepsilon_{0} \frac{\partial \vec{E}}{\partial t}=\operatorname{rot} \vec{H}, \\
\operatorname{div} \vec{H}=0
\end{gathered}
$$

Introduced thus magnetic field is vortex. Comparing (12.9) and (12.10) we obtain:

$$
\frac{\partial H_{z}(x, t)}{\partial x}=\frac{e^{2} \omega \sin \alpha}{4 \pi c^{2} m x} E_{y 0}^{\prime} \cos \omega\left(t-\frac{x}{c}\right) .
$$

Integrating this equation on the coordinate, we find the value of the magnetic field

$$
H_{z}(x, t)=\frac{e^{2} \sin \alpha}{4 \pi c m x} E_{y 0}^{\prime} \sin \omega\left(t-\frac{x}{c}\right) .
$$

Thus, Eqs. (12.8), (12.9) and (12.11) can be named the laws of electro-electrical induction, since. they give the direct coupling between the electric fields, applied to the charge, and by fields and by currents induced by this charge in its environment. Charge itself comes in the role of the transformer, which ensures this reradiation. The magnetic field, which can be calculated with the aid of Eq. (12.11), is directed normally both toward the electric field and toward the direction of propagation, and their relation at each point of the space is equal 


$$
\frac{E_{y}(x, t)}{H_{Z}(x, t)}=\frac{1}{\varepsilon_{0} c}=\sqrt{\frac{\mu_{0}}{\varepsilon_{0}}}=Z,
$$

where $Z$ is wave drag of free space.

The combination of electrical and magnetic wave is called the electromagnetic wave

Wave drag determines the active power of losses on the single area, located normal to the direction of propagation of the wave:

$$
P=\frac{1}{2} Z E_{y 0}^{2}
$$

Therefore electrocurent wave, crossing this area, transfers through it the power, determined by the data by equation, which is located in accordance with Poynting theorem about the power flux of electromagnetic wave. Therefore, for finding all parameters, which characterize wave process, it is sufficient examination only of electrocurent wave and knowledge of the wave drag of space. In this case it is in no way compulsory to introduce this concept as magnetic field and its vector potential, although there is nothing illegal in this. In this setting of the equations, obtained for the electrical and magnetic field, they completely satisfy Helmholtz theorem. This theorem says, that any single-valued and continuous vectorial field $\vec{F}$, which turns into zero at infinity, can be represented uniquely as the sum of the gradient of a certain scalar function of and rotor of a certain vector function, whose divergence is equal to zero:

$$
\begin{aligned}
& \vec{F}=\operatorname{grad} \varphi+\operatorname{rot} \vec{C}, \\
& \operatorname{div} \vec{C}=0 .
\end{aligned}
$$

Consequently, must exist clear separation fields on to the gradient and the vortex. It is evident that in the expressions, obtained for those induced field on, this separation is located. Electric fields have gradient nature, and magnetic is vortex field.

Thus, the construction of electrodynamics should have been begun from the acknowledgement of the dependence of scalar potential on the speed. But nature very deeply hides its secrets, and in order to come to this simple conclusion, it was necessary to pass way by length almost into two centuries. The grit, which so harmoniously were erected around the magnet poles, in a straight manner indicated the presence of some power field on potential nature, but to this they did not turn attention; therefore it turned out that all examined only tip of the iceberg, whose substantial part remained invisible of almost two hundred years.

Taking into account entire aforesaid one should assume that at the basis of the overwhelming majority of static and dynamic phenomena at the electrodynamics only one formula (19.1), which assumes the dependence of the scalar potential of charge on the speed, lies. From this law follows and static interaction of charges, and the laws of their power interaction in the case of mutual motion, and the emission laws and scattering, the phase aberration of electromagnetic waves, and the transverse Doppler effect. After entire aforesaid it is possible to remove construction forests, such as magnetic field and magnetic vector potential, which do not allow here already almost two hundred years to see the building of electrodynamics in entire its sublimity and beauty.
Let us point out that one of the fundamental equations of induction (12.4) could be obtained directly from the Ampere law, still long before appeared Maxwell equations. The Ampere law, expressed in the vector form, determines magnetic field at the point

$$
\vec{H}=\frac{1}{4 \pi} \int \frac{I d \vec{l} \times \vec{r}}{r^{3}}
$$

where $I$ is current in the element $d \vec{l}, \vec{r}$ is vector, directed from $d \vec{l}$ to the point $x, y, z$.

It is possible to show that

$$
\frac{[d \vec{l} \vec{r}]}{r^{3}}=\operatorname{grad}\left(\frac{1}{r}\right) \times d \vec{l}
$$

and, besides the fact that

$$
\operatorname{grad}\left(\frac{1}{r}\right) \times d \vec{l}=\operatorname{rot}\left(\frac{d \vec{l}}{r}\right)-\frac{1}{r} \operatorname{rot} d \vec{l} .
$$

but the rotor $d \vec{l}$ is equal to zero and therefore is final

$$
\vec{H}=\operatorname{rot} \int I\left(\frac{d \vec{l}}{4 \pi r}\right)=\operatorname{rot} \vec{A}_{H},
$$

where

$$
\vec{A}_{H}=\int I\left(\frac{d \vec{l}}{4 \pi r}\right)
$$

The remarkable property of this expression is that that the vector potential depends from the distance to the observation point as $\frac{1}{r}$. Specifically, this property makes it possible to obtain emission laws.

Since $I=g v$, where $g$ linear charge, from Eq. (12.12) we obtain:

$$
\vec{A}_{H}=\int \frac{g v d \vec{l}}{4 \pi r} .
$$

For the single charge $e$ this equation takes the form:

$$
\vec{A}_{H}=\frac{e \vec{v}}{4 \pi r},
$$

and since

$$
\vec{E}=-\mu \frac{\partial \vec{A}}{\partial t}
$$

that

$$
\vec{E}=-\mu \int \frac{g \frac{\partial v}{\partial t} d \vec{l}}{4 \pi r}=-\mu \int \frac{g a d \vec{l}}{4 \pi r}
$$

where $a$ is acceleration of charge.

This equation appears as follows for the single charge:

$$
\vec{E}=-\frac{\mu e \vec{a}}{4 \pi r} .
$$

If we in Eqs. (12.13) and (12.14) consider that the potentials are extended with the final speed and to 
consider the delay $\left(t-\frac{r}{c}\right)$, and assuming, these equations will take the form:

$$
\begin{gathered}
\vec{E}=-\mu \int \frac{g a\left(t-\frac{r}{c}\right) d \vec{l}}{4 \pi r}=-\int \frac{g a\left(t-\frac{r}{c}\right) d \vec{l}}{4 \pi \varepsilon_{0} c^{2} r} \\
\vec{E}=-\frac{e \vec{a}\left(t-\frac{r}{c}\right)}{4 \pi \varepsilon_{0} c^{2} r} .
\end{gathered}
$$

where the equations (12.15) and (12.16) represent wave equations. Let us note that these equations - this solution of Maxwell equations, but in this case they are obtained directly from the Ampere law, not at all coming running to Maxwell equations. To there remains only present the question, why electrodynamics in its time is not banal by this method?

\section{Scalar-vector Potential and the Formation of Electrical Fields on the Inductions also of the Magnetic Vector Potential}

Earlier has already been indicated that solution of problems interactions of the moving charges in the classical electrodynamics are solved by the introduction of the magnetic field or vector potential, which are fields by mediators. To the moving or fixed charge action of force can render only electric field. Therefore natural question arises, and it is not possible whether to establish the laws of direct action, passing fields the mediators, who would give answer about the direct interaction of the moving and fixed charges. This approach would immediately give answer, also, about sources and places of the application of force of action and reaction. Let us show that application of scalar- vector potential gives the possibility to establish the straight laws of the induction, when directly the properties of the moving charge without the participation of any auxiliary field on they give the possibility to calculate the electrical induction fields, generated by the moving charge.

Let us examine the diagram of the propagation of current and voltage in the section of the long line, represented in Figure 10. Let us assume that in the time $t$ voltage on incoming line, changing according to the linear law, reached its nominal value $U$. This period of time we will call the front of wave (Fig. 10). In the long line this front occupies the section of the long $z_{1}$. Let us explain, from where are taken those electric fields, which it forces the charges, located near the conductors of line, to move in the direction opposite to the direction of the motion of charges in line itself. In the section $z_{1}$ proceeds the acceleration of charges from their zero speed (more to the right the section $z_{1}$ ) to the value of speed, determined by the equation

$$
v=\sqrt{\frac{2 e U}{m}},
$$

where $e$ and $m$ are charge and the mass of current carriers, $U$ is voltage drop across the section $z_{1}$. Then the dependence of the speed of current carriers on the coordinate will take the form:

$$
v^{2}(z)=\frac{2 e}{m} \frac{\partial U}{\partial z} z .
$$

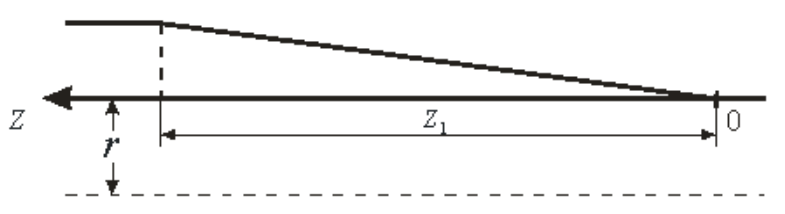

Figure 10. Current wavefront, which is extended in the long line

Since we accepted the linear dependence of stress from the time on incoming line, the equality occurs

$$
\frac{\partial U}{\partial z}=\frac{U}{z_{1}}=E_{Z},
$$

where $E_{z}$ is field strength, which accelerates charges in the section $z_{1}$. Consequently, Eq. (13.1) it is possible to rewrite

$$
v^{2}(z)=\frac{2 e}{m} E_{z} z .
$$

Using for the value of scalar- vector potential Eq. (9.4), let us calculate it as the function $z$ on a certain distance $r$ from the line

$\varphi(z)=\frac{e}{4 \pi \varepsilon_{0} r}\left(1+\frac{1}{2} \frac{v^{2}(z)}{c^{2}}\right)=\frac{e}{4 \pi \varepsilon_{0} r}\left(1+\frac{e E_{z} z}{m c^{2}}\right)$.

For the record Eq. (13.2) are used only first two members of the expansion of hyperbolic cosine in series.

Using the eqution $E=-\operatorname{grad} \varphi$, and differentiating Eq. (13.2) on $z$, we obtain

$$
E_{z}{ }^{\prime}=-\frac{e^{2} E_{z}}{4 \pi \varepsilon_{0} r m c^{2}},
$$

where $E_{z}{ }^{\prime}$ is the electric field, induced at a distance $r$ from the conductor of line. Near $E$ there is a prime in connection with the fact that calculated field it moves along the conductor of line with the speed of light. This field acts on the charges, which surround line, forcing them to move in the opposite direction with respect to those charges, which move in the line. The acceleration of charge is determined by the equation $a_{z}=\frac{e E_{Z}}{m}$. Taking this into account from (13.3) we obtain

$$
E_{z}{ }^{\prime}=-\frac{e a_{z}}{4 \pi \varepsilon_{0} r c^{2}} .
$$

Thus, the charges, accelerated in the section of the line $z_{1}$, induce at a distance $r$ from this section the electric field, determined by Eq. (13.4). Direction of this field conversely to field, applied to the accelerated charges. Thus, is obtained the law of direct action, which indicates what electric fields generate around themselves the 
charges, accelerated in the conductor. This law can be called the law of electro-electrical induction, since it, passing fields mediators (magnetic field or vector potential), gives straight answer to what electric fields the moving electric charge generates around itself. This law gives also answer about the place of the application of force of interaction between the charges. Specifically, this equation we must consider as the fundamental law of induction, since specifically, it establishes the reason for the appearance of induction electrical field on around the moving charge. In what the difference between the proposed approach and that previously existing consists. Earlier we said that the moving charge generates vector potential, and the already changing vector potential generates electric field. The equation (13.4) gives the possibility to exclude this intermediate operation and to pass directly from the properties of the moving charge to the induction fields. Let us show that equation it follows from this and the introduced earlier phenomenologically vector potential, and, therefore, also magnetic field. Since the connection between the vector potential and the electric field is determined by Eq. (2.3), equality (13.4) it is possible to rewrite

$$
E_{z}{ }^{\prime}=-\frac{e}{4 \pi \varepsilon_{0} r c^{2}} \frac{\partial v_{z}}{\partial t}=-\mu \frac{\partial A_{H}}{\partial t},
$$

and further, integrating by the time, we obtain

$$
A_{H}=\frac{e v_{Z}}{4 \pi r} .
$$

This equation corresponds to the determination of vector potential. It is now evident that the vector potential is the direct consequence of the dependence of the scalar potential of charge on the speed. The introduction also of vector potential and of magnetic field this is the useful mathematical device, which makes it possible to simplify the solution of number of electrodynamic problems, however, one should remember that by fundamentals the introduction of these fields on it appears scalar- vector potential.

\section{Conclusion}

We passed large way on the examination of the problems of those accumulating in the contemporary electrodynamics, and, in spite of that which to this science is already more than 200 years, these problems remained still sufficiently much. For the duration entire of the period in the electrodynamics indicated primary attention was paid to the electrical and magnetic fields, and this concept as magnetic vector potential remained in the shadow. The analysis, carried out in this article, showed that the magnetic vector potential is one of the most important concepts of electrodynamics. But physical nature of this potential before the appearance of articles $[5,13,14,15,18]$ was not clear. Now we can draw that scenario, on which must be developed the electrodynamics, if the role of magnetic vector potential, was realized in the early stages of its development, and then if at that time was possible to understand its physical nature.

The Ampere law, expressed in the vector form, determines magnetic field at the point

$$
\vec{H}=\frac{1}{4 \pi} \int \frac{I d \vec{l} \times \vec{r}}{r^{3}},
$$

where $I$ is current in the element $d \vec{l}, \vec{r}$ is vector, directed from $d \vec{l}$ to the point $x, y, z$.

It is possible to show that

$$
\frac{[d \vec{l} \vec{r}]}{r^{3}}=\operatorname{grad}\left(\frac{1}{r}\right) \times d \vec{l} .
$$

But also, that

$$
\operatorname{grad}\left(\frac{1}{r}\right) \times d \vec{l}=\operatorname{rot}\left(\frac{d \vec{l}}{r}\right)-\frac{1}{r} \operatorname{rot} d \vec{l},
$$

but the rotor of $d \vec{l}$ is equal to zero and therefore is final

$$
\vec{H}=\operatorname{rot} \int I\left(\frac{d \vec{l}}{4 \pi r}\right)=\operatorname{rot} \vec{A}_{H},
$$

where

$$
\vec{A}_{H}=\int I\left(\frac{d \vec{l}}{4 \pi r}\right)
$$

The remarkable property of this expression is that that the vector potential depends from the distance to the observation point as $\frac{1}{r}$. Specifically, this property makes it possible to obtain emission laws.

Since $I=g v$, where $g$ is linear charge, from Eq. (12.12) we obtain:

$$
\vec{A}_{H}=\int \frac{g v d \vec{l}}{4 \pi r} .
$$

For the single charge of this equation takes the form:

$$
\vec{A}_{H}=\frac{e \vec{v}}{4 \pi r},
$$

and since

$$
\vec{E}=-\mu \frac{\partial \vec{A}}{\partial t}
$$

the knowledge of vector potential, its time derivatives and on the coordinates makes it possible to find electrical and magnetic field.

Equation

$$
\operatorname{rot} \operatorname{rot} \vec{A}_{H}=\vec{j}\left(\vec{A}_{H}\right),
$$

obtained in the second paragraph indicates that $\operatorname{rot} \operatorname{rot} \vec{A}_{H}$ is the functional of current density, which for the different media is determined by the following equations:

For the free space and the dielectrics:

$$
\operatorname{rot} \operatorname{rot} \vec{A}_{H}=-\mu \varepsilon \frac{\partial^{2} \vec{A}_{H}}{\partial t^{2}} .
$$

For the case of fulfilling Ohm's law:

$$
\operatorname{rot} \operatorname{rot} \vec{A}_{H}=-\sigma \mu \frac{\partial \vec{A}_{H}}{\partial t} .
$$


For the conductors, in which be absent the ohmic losses:

$$
\operatorname{rot} \operatorname{rot} \vec{A}_{H}=-\frac{\mu}{L_{k}} \vec{A}_{H} \text {. }
$$

The equations (14.1-14.3) are wave equations and make it possible to obtain the laws of the propagation of vector potential in different media.

Having Eqs. (14.1-14.3) it is possible to immediately obtain wave equations for the electrical and magnetic field. Differentiate on the time from both parts of these equations, we obtain:

$$
\begin{aligned}
& \operatorname{rot} \operatorname{rot} \vec{E}=-\mu \varepsilon \frac{\partial^{2} \vec{E}}{\partial t^{2}}, \\
& \operatorname{rot} \operatorname{rot} \vec{E}=-\sigma \mu \frac{\partial \vec{E}}{\partial t}, \\
& \operatorname{rot} \operatorname{rot} \vec{E}=-\frac{\mu}{L_{k}} \vec{E}
\end{aligned}
$$

After taking rotor from both parts of equations (14.1-14.3), we obtain wave equations for the magnetic field for the media indicated:

$$
\begin{aligned}
& \operatorname{rot} \operatorname{rot} \vec{H}=-\mu \varepsilon \frac{\partial^{2} \vec{H}}{\partial t^{2}}, \\
& \operatorname{rot} \operatorname{rot} \vec{H}=-\sigma \mu \frac{\partial \vec{H}}{\partial t}, \\
& \operatorname{rot} \operatorname{rot} \vec{H}=-\frac{\mu}{L_{k}} \vec{H}
\end{aligned}
$$

With this approach Maxwell equations no longer are necessary to us, since given equations give the possibility to examine wave processes in all media indicated.

But vector potential makes it possible to solve not only wave problems. With its aid, as shown in the second paragraph, can be solved the problems of power interaction of the current-carrying systems, which up to now were solved with the aid of the postulate about the Lorentz force.

Moreover, the use of vector potential makes it possible to write down the complete law of induction (2.7) without any withdrawals and exceptions. And precisely vector potential makes electrodynamics with one-piece and united science. But physical nature of this potential before the appearance of articles $[5,13,14,15,18]$ was not clear. This articl only details those ideas, which are expressed in these articles. The obtained results change the ideological basis of classical electrodynamics, indicating that the substantial part of the observed in the electrodynamics dynamic phenomena, this by the consequences of this dependence. Certainly, the adoption of this concept is critical step. But this step is transparent and intelligible from a physical point of view. Indeed the main parameter of charge are those energy characteristics, which it possesses and how it influences the surrounding charges not only in the static position, but also during its motion. The dependence of scalar potential on the speed leads to the fact that in its environments are generated the electric fields, to reverse fields, that accelerate charge itself. Such dynamic properties of charge allow instead of two symmetrical laws of magnetoelectric and electromagnetic induction to introduce one law of electro-electrical induction, which is the fundamental law of induction. This method gives the possibility to directly solve all problems of induction and emission, without resorting to the application of such field on mediators as vector potential and magnetic field. This approach makes it possible to explain the origin of the forces of interaction between the current carrying systems. The introduction of scalarvector potential explains a number of the phenomena, such as phase aberration and the transverse Doppler effect, which earlier in the classical electrodynamics an explanation did not have.

Up to now in the classical electrodynamics existed two not connected with each other of division. From one side this of Maxwell equation, and from which follow wave equations for the electromagnetic field on, while from other side this of the equations, which determine power interaction of the current carrying systems. For explaining this phenomenon the postulate about the Lorentz force was introduced. Introduction to the dependence of the scalar potential of charge on the speed mutually connects these with those not connected divisions, and classical electrodynamics takes the form of the ordered united science, which has united ideological basis.

Article shows that together with the fundamental parameters $\varepsilon \varepsilon_{0}$ and $\mu \mu_{0}$, which characterize the specific forms of energy, accumulated or transferred on the material media, namely: electrical and magnetic energy; there are two additional fundamental material parameters: the kinetic inductance $L_{k}$ and the kinetic capacity $C_{k}$. With these parameters are connected two forms of energy, namely: kinetic and potential, which can be accumulated or be transferred in the material media. If, the parameter, were sometimes and used with the description of some physical phenomena, for example, in the superconductors, then there were no, before the appearance of article [5], known about existence. Use of all four parameters $\varepsilon \varepsilon_{0}$, $\mu \mu_{0}, L_{k}$ and $C_{k}$ gives the possibility to solve the problems of accumulation and propagation of energy in the material media. Earlier it was always considered that in the material media the electromagnetic waves are propagated and only these waves transfer energy. However, this approach is insufficient to account for all forms of the energy, accumulated and transferred by fields and currents in the material media. In actuality in the material media are propagated the magnetoelectrokinetic or electromagnetopotential waves, in which the part of the energy is accumulated and is transferred purely mechanically. Resonances in the material media also bear their specific character. In contrast to the electromagnetic resonances in the locked planes, when the energy exchange occurs between the magnetic and electric fields, in the material media there are two forms of resonances. This is the electrokinetic resonance, when electric field energy is converted into the kinetic energy of charge carriers and vice versa, and magnetic fields on material media be absent. The second resonance it is possible to name magnetopotential resonance, when the potential energy, accumulated in the precessional motion of magnetic moments, can return into the external space at the frequency of precession. 
Should be focused attention also to the fact that the physical interpretation of some mathematical concepts, which concern electrodynamic processes, they require the specific caution. So it is mathematically very simple of two, not depending on the frequency physical quantities, to design the mathematical symbol, which will depend on frequency. Specifically, this occurred, when the concepts of the dispersion of dielectric and magnetic constant were introduced. However, it turned out that such concepts as the dispersion of these values, physically [neobosnovanny], although the parameter $\varepsilon^{*}(\omega)$ is convenient for the mathematical description of the processes, which exist in the material media.

We are the witnesses of the phenomenon, when mathematics bore the new physical parameter, which in nature there does not exist. And, the most interesting consists in the fact that all of physics into existence of this parameter believed and many believe to the these rapids. The discussion deals with the frequency-dependent dielectric constant. This physical parameter in nature there does not exist. How did arise this error? It is known that there is a dispersion of electromagnetic waves with their passage through the material media. Here all began to think that this dispersion was generated by the dispersion of dielectric constant. Occurs everything entirely not thus. The dispersion of electromagnetic waves is the consequence not of the dispersion of dielectric constant, but the dispersion of the phase speed of electromagnetic waves. Immediately several parameters independent from the frequency form this dispersion. For the plasma the dielectric constant of vacuum and the kinetic inductance of charge carriers, which present plasma, are them. In the dielectrics this process somewhat more complex, because, together with the dielectric constant of vacuum and the kinetic inductance of bound charges, in this process participate the polarization or orientational properties of the electric dipoles, existing in the dielectric. Consequently, the dispersion of electromagnetic waves, which is observed with their propagation in the dielectrics, is connected not with the dispersion of the nonexistent parameter, which was considered the frequency-dependent dielectric constant, but with the dispersion of reactive dielectric conductance.

But if assertion about the presence of dispersion in the dielectric and magnetic constant can be to a certain degree considered systematic error, then in the electrodynamics of continuous media there are errors also of more fundamental nature. This is that case, when in conducting media at the microscopic level is introduced polarization vector similarly, as is done in dielectrics [7]. This is gross physical error, since polarization vector in the conducting structures at the microscopic level be it cannot, since in the conductors the charges are free, and the electric dipoles do not can to form. As a result such systematic and physical errors unnoticed proved to be the circumstance that in the nonmagnetized confined plasma, together with the longitudinal Langmuir resonance, it can occur and transverse plasma resonance, the frequencies in these resonances coinciding, i.e., they are degenerate. But this means that is passed the entire scientific and technical direction, which has great applied value.

\section{References}

[1] R. Feynman, R. Leighton, M. Sends, Feynman lectures on physics, M:, Mir, Vol.6, 1977.

[2] V.V. Nicolsky, T.I. Nicolskaya, Electrodynamics and propagation of radio waves, Moscow, Nauka,1989.

[3] J. Jackson, Classical Electrodynamics, Mir, Moscow, 1965.

[4] F.F. Mende, A.I. Spitsyn, Surface impedance in superconductors, Kiev, Naukova Dumka, 1985.

[5] F. F. Mende, On refinement of equations of electromagnetic induction, - Kharkov, deposited in VINITI, No 774-B88 Dep., 1988.

[6] A. F. Aleksandrov, L.S. Bogdankevich, A. A. Rukhdze, Oscillations and waves in plasma media, Moscow University Publishers, 1990.

[7] L. D. Landau, E.M. Lifshits, Electrodynamics of continuous media. Moscow. 1973.

[8] V. L. Ginzburg, The propagation of electromagnetic waves in a plasma. - M .: Science. 1967.

[9] A. I. Akhiezer, Plasma Physics Nauka, Moscow, 1974.

[10] L. A. Artsimovich As every physicist should know about plasma. M .: Atomizdat, 1976.

[11] F. F. Mende, Role and place of the kinetic inductance of charges in classical electrodynamics, Engineering Physics, №11, 2012.

[12] F. F. Mende, Transverse Plasma Resonans Mode in an Nonmagnetized Plasma and Its Practical Applications, American Journal of Electrical and Electronic Engineering, Vol. 2, No. 5, (2014), 152-158.

[13] F.F. Mende, On refinement of certain laws of classical electrodynamics, arXiv.org/abs/physics/0402084.

[14] F. F. Mende, Are there errors in modern physics. Kharkov, Constant, 2003.

[15] F. F. Mende, Conception of the scalar-vector potential in contemporary electrodynamics, arXiv.org/abs/physics/0506083.

[16] F. F. Mende, Transversal plasma resonance in a nonmagnetized plasma and possibilities of practical employment of it, arXiv.org/abs/physics/0506081.

[17] F. F. Mende, Consistent electrodynamics and the threat of nuclear Space terrorism. Kharkov NTMT, 2008.

[18] F. F. Mende New electrodynamics. Revolution in the modern physics. Kharkov, NTMT, 2012.

[19] F. F. Mende, Electric kinetic capacity. Engineering Physics, №3, 2013.

[20] V. V.Nicolsky, T. I. Nicolskaya, Electrodynamics and propagation of radio waves, Moscow, Nauka, 1989.

[21] W. F. Edwards, C. S. Kenyon, D. K. Lemon, Continuing investigation into possible electric arising from steady conduction current, Phys. Rev. D 14, 922, 1976.

[22] Roser W.G.V. Second-Order Electric Field due to a Conducting Curent. American Journal of Physics, 1962, v. 30, №7, p. 509-511.

[23] Don A. Baker. Second-Order Electric Field due to a Conducting Curent. American Journal of Physics, 1964, v.32, № 2, p. 153-157.

[24] F. F. Mende, On secondary electric fields excited at direct currents flowing through superconductors. - Kharkov, deposited in VINITI 05.11.92. No. 3182-B92. Dep., 1992.

[25] F. F. Mende, Experimental corroboration and theoretical interpretation of dependence of charge velocity on DC flow velocity through superconductors. Proceedings International Conference "Physics in Ukraine", Kiev 22-27 June, 1993.

[26] F. F. Mende, A New Tipe of Contact Potential Difference and Electrification of Superconducting Coils and Tori, American Journal of Electrical and Electronic Engineering, Vol. 2, No. 5, 2014, 146-151. 